\title{
Théologiques
}

\section{La Bible relue par les animaux}

\section{Olivette Genest}

Volume 10, numéro 1, printemps 2002

Les animaux dans la conscience humaine

URI : https://id.erudit.org/iderudit/008159ar

DOI : https://doi.org/10.7202/008159ar

Aller au sommaire du numéro

Éditeur(s)

Faculté de théologie de l'Université de Montréal

ISSN

1188-7109 (imprimé)

1492-1413 (numérique)

Découvrir la revue

Citer cet article

Genest, O. (2002). La Bible relue par les animaux. Théologiques, 10(1), 131-177. https://doi.org/10.7202/008159ar

\section{Résumé de l'article}

Les discussions éthiques autour du comportement à adopter envers les animaux recourent souvent à des passages bibliques. Devant des réponses divergentes, l'auteure tente de faire le point sur leur statut dans la Bible, Ancien et Nouveau Testament. L'enquête porte sur les passages les plus significatifs : création, déluge, classification selon le pur et l’impur, rôle dans le système sacrificiel d'Israël et les cultes du monde gréco-romain où se répand le christianisme naissant. La conclusion soulève brièvement la question de la pertinence du recours à la Bible et de sa normativité sur ce point. d'utilisation que vous pouvez consulter en ligne.

https://apropos.erudit.org/fr/usagers/politique-dutilisation/ 


\title{
La Bible relue par les animaux ${ }^{1}$
}

\author{
Olivette Genest \\ Professeure émérite \\ Faculté de théologie \\ Université de Montréal
}

\section{Introduction}

Les animaux entrent dans la Bible par la première page et y demeurent jusqu'à la dernière. Y pullulent des animaux du monde naturel, tels que nous les connaissons, et des animaux métaphoriques et symboliques issus de l'imagination des scribes inspirés. La relecture par cette population animalière proposée dans le titre de l'article n'entend déboucher ni sur un bestiaire ni sur une tératologie fantastique, malgré l'intérêt du projet déjà tenté ailleurs. Elle ne donnera pas non plus la parole et la plume à des animaux-à-lunettes pour une réécriture animale de l'épopée biblique, malgré la fécondité alléchante de ce procédé littéraire déjà démontrée également chez les virtuoses de la fable du type de George Orwell dans Animal Farm et de Clifford Simak dans Demain les chiens (The City), par exemple ${ }^{2}$.

Cette relecture par le filon des animaux relève plutôt du dossier d'information à l'usage de qui se tourne vers la Bible pour éclairer

1. À la mémoire de François Martin, le théologien sémioticien qui découvrit avec émerveillement, sur le Mont-Royal, des tamias rayés, nos "petits-suisses » québécois. Ces écureuils enjolivés n'étaient donc pas une création de Walt Disney mais bien des créatures du Père Éternel!

2. G. ORWELL, La ferme des animaux; Animal Farm / préface et notes par Y. Yvinec / trad. par J. Quéval (Folio bilingue 38), Paris, Gallimard, 1994 (anglais 1945); C.D. Simak, Demain les chiens / trad. par J. Rosenthal (Science-fiction 373), Paris, J'ai lu, 1975 (anglais 1952). 
les questions éthiques, nombreuses dans l'air du temps, au sujet de notre comportement envers nos co-locataires de cette planète, nos si proches parents. À cause des dimensions et du Livre et de la présence animale entre ses pages, la discussion de la pertinence de la Bible, de sa normativité à ce sujet et de la nature de ce que serait un comportement spécifiquement chrétien envers les bêtes devra être renvoyée à l'usager.

Pour préserver des réponses par choix de versets extraits de leurs contextes, cette étude panoramique s'attachera à la mise en discours de la gent animale dans la Bible chrétienne, c'est-à-dire dans le corpus Ancien et Nouveau Testament, abordé comme œuvre littéraire, par l'angle d'accès du lecteur non spécialisé, croyant ou féru de littérature ancienne. Nous y suivrons à la trace les animaux dans leur habitat biblique, par bonds d'antilope entre les passages les plus significatifs. L'observation se limitera à la classe des animaux "réels ", conservant à la périphérie du champ visuel délimité leurs congénères imaginaires.

À l'intérieur de ces frontières et conventions de départ, les animaux de la Bible ne sont-ils que les figurants de service du décor de toute étendue sauvage et de toute société terrienne? Ou des acteurs d'un récit qui leur attribue un sort, une fortune dans l'histoire du peuple de Dieu? Dans un livre occupé de salut, quel est le leur, si tant il en est qu'ils seraient en manque de salut? Ces questions-là appartiennent à cette étude-ci. À qui voudra poursuivre la réflexion et la casuistique du quotidien, elle est forcée de renvoyer les interrogations subséquentes. Si les animaux sont les partenaires de la race humaine, devrait-elle l'être pour eux? Sont-ils notre prochain? Et qui est le prochain de l'autre, selon la formulation inversée par Jésus dans la parabole du bon Samaritain (Lc 10,36)? 


\section{PETITE ZOOLOGIE BIBLIQUE}

\section{Le Seigneur des animaux - Genèse 1, 2 et 3}

\subsection{Genèse 1}

«Il y eut un soir, il y eut un matin: jour premier. » Portail d'entrée de toute la Bible, le poème cosmogonique de Genèse 1 répétera sept fois la symbolique démarcation temporelle de la semaine qui lance l'univers. Chaque jour trouvera sa complétude dans l'expression de la satisfaction divine: "Et Dieu vit que cela était bon, très bon."

La beauté des images et le rythme solennel masquent cependant, à la première lecture, l'ordonnance systématique qui préside à la création. Dans ce chaos originel fait de ténèbres et de grandes eaux sans frontières, un Dieu méthodique, verset après verset, inscrit par sa parole créatrice cadre, contenants, contenus et destins. Les ténèbres reçoivent la lumière et son alternance avec elles qui auront nom jour et nuit; les eaux, une cloison séparatrice en forme de voûte appelée ciel; les eaux sous le ciel, une concentration nommée mers qui dégage une masse sèche désignée terre. Les lieux sont en place, avec leur nom propre; il reste à les aménager. La terre se couvre d'herbes portant semence selon leurs espèces et d'arbres fruitiers produisant des fruits également dotés de leur semence spécifique, donc de leur moyen de reproduction et de continuité. Au ciel, s'allument de grands luminaires pour éclairer la nuit terrestre.

Les habitats préparés attendent leur peuplement. Au cinquième jour du grand œuvre, la parole convoque le vivant. Ouvrant la série, l'animal apparaît le premier, portant comme sien propre le nom de cette nouvelle catégorie. Dans l'hébreu du texte, animal se dit: nefesh bayyāh, littéralement "être vivant", tout comme l'humain et à la différence de la plante et des astres ${ }^{3}$. À leur appel à l'existence, et selon l'espèce qui leur a été dévolue, ces «êtres vivants » envahissent les eaux puis le ciel, poissons, grands monstres marins, toutes les

3. En français et en anglais, nous oublions à l'usage que le mot «animal » vient du latin animalis, de anima ou âme. 
créatures qui glissent sur les sentiers de la mer, toute la gent ailée qui vole contre le firmament.

Au sixième jour, la terre se remplit à son tour des vivants adaptés à cet espace, bestiaux, petites bêtes du sol et bêtes sauvages selon leur espèce (v. 24), rangés ensuite en: bêtes sauvages, bestiaux, petites bêtes du sol (v. 25), c'est-à-dire des plus gros animaux aux plus petits. Ce Dieu à la toute-puissance débordante aime tout de même l'ordre et le rythme régulier dans le travail. Par addition d'éléments connexes, il a peu à peu dressé la scène pour l'émergence du vivant qui lui ressemblera, l'humain, du nom collectif `ādām, dérivé de ‘adàmāh, sol ou glaise, qu'on pourrait traduire littéralement: le terreux. La dignité d'image de Dieu ne rompt pas la parenté avec l'animal terrestre à propos duquel Dieu avait dit: "Que la terre produise des êtres vivants...» (v. 24). S'ajoute pour l'humain la mention de la distinction mâle et femelle (v. 27), et le passage des pronoms du singulier au pluriel témoigne de la création simultanée de l'homme et de la femme: "...à l'image de Dieu, il le créa, mâle et femelle il les créa. " À l'issue d'une série d'êtres qui auront tous fait irruption dans la vie en grand nombre et en grande diversité, il ne nous semble pas exclu de pouvoir lire en Gn 1 la création de plusieurs couples à la racine du genre humain, ce qui ne sera manifestement pas le cas en Gn 2.

Tout déborde de vitalité dans ce poème heureux. Tout y foisonne, pullule, prolifère dès le départ (ce sont les verbes du texte). Les destinées attribuées aux différentes classes de vivants iront dans le même sens. Aux créatures marines, aux oiseaux, comme aux humains, Dieu dit en les bénissant: "Soyez féconds, multipliez-vous, remplissez votre espace vital! »Curieusement, les bêtes sauvages, le bétail et les petits animaux n'entendent pas la même consigne. Peutêtre parce qu'elle dépend du couple anthropoïde qui doit régner sur eux...et qui se pose encore la question du juste rapport à adopter envers ces bêtes! Une dernière disposition du Créateur donne aux humains, aux bêtes sauvages, aux oiseaux et petits animaux, à toute nefesh hayyāh, les herbes et les arbres fruitiers en nourriture. Les êtres vivants seront donc végétariens. Échappent à la reprise de la 
liste des créatures, plusieurs fois répétée en Gn 1, les poissons de la mer et les bestiaux...dont les humains sont chargés. À la fin de la semaine inaugurale du monde, comme à la fin de chaque jour, Dieu regarde son œuvre. Ces animaux du sixième jour, «Dieu vit que cela était bon ». L'ensemble de tout ce qu'il avait fait d'ailleurs, «Dieu vit que cela était vraiment très bon ». Et, dans le fil du temps, l'espace de ce septième jour naissant, celui de l'arrêt de ses activités et de son contentement, Dieu le mit à part, ce qui est le premier sens de consacrer, de sacrer.

\subsection{Genèse 2}

La suite de la Genèse ne s'engage pas dans l'histoire de la deuxième semaine, mais reprend un deuxième récit de la création (Gn 2,4-24). De structure narrative et de facture stylistique différentes, il n'en continue pas moins à construire les catégories de base du monde ${ }^{4}$. L'Élohîm de Gn 1 était passé du spatio-temporel au végétal, puis à l'animal et à l'humain. Le Yahvé ${ }^{2}$ de Gn 2 modifie la séquence, procédant de l'humain indifférencié à l'espace, au végétal, à l'animal et à l'humain homme et femme. D'une terre stérile, humectée par des sources de surface, le potier divin modèle d'abord la créature-du-sol, le ${ }^{a} \bar{a} \bar{a} m$, lui insuffle l'haleine de vie (nе̌shāmāh) et il devient nefesh bayyāh, être vivant. L'apparition du jardinier rend le jardin possible et la croissance de «tout arbre beau à voir et bon à manger », opposé

4. Gn 1 et Gn 2 proviennent de traditions différentes que les travaux des exégètes datent respectivement du vi ${ }^{\mathrm{e}}$ A.C. (grosso modo de la déportation à Babylone, soit de la prise de Jérusalem en - 586 à l'édit de libération de Cyrus en - 538) et du viII ${ }^{\mathrm{e}}$ A.C., du temps du roi Ézéchias et du prophète Isaïe. En réalité, c’est Gn 1 qui «continue» Gn 2. Nous avons choisi d'adopter l'angle du lecteur, l'ordre de lecture. Rétablir celui de l'ordre d'écriture donnerait une tout autre Bible, sans affecter, à mon avis, les animaux qui nous intéressent.

5. En Gn 1, l'acteur Dieu se nomme Élohîm, en Gn 2 et 3, Yahvé-Élohîm sous la plume du narrateur et Élohîm quand le serpent et la femme parlent de lui. Élohîm est un nom collectif, pluriel, celui d'une catégorie de vivants, les dieux; appliqué à Yahvé, il commande cependant des verbes au singulier. Yahvé est un nom propre, donc singulier, celui de ce dieu que la Bible pose comme le Dieu unique. 
aux arbustes des champs et à l'herbe des champs. Spatialement, le texte passe de la steppe au jardin, au milieu du jardin où deux arbres transcendent l'ordre végétal: l'arbre de vie et celui de la connaissance du bon et du mauvais. Une restriction entre au paradis: " tu mangeras de tout, sauf de ces deux arbres. » Dans l'horizon de la luxuriance de vie pointe la possibilité de la mort.

Malgré la séduction des énigmes miroitant au détour de chaque verset, la construction littéraire de l'acteur «animal » nous occupera désormais au premier chef. En Gn 2, Yahvé crée les animaux expressément pour contrer la solitude du 'ādām et pour lui être une aide dans sa charge de gérant et de gardien du jardin (en hébreu, 'èzer) une aide qui lui soit appareillée "comme un vis-à-vis", ajoute le texte. Il les modèle également du sol et les amène au ${ }^{\circ} \bar{a} d \bar{a} m$ (première procession des animaux dans la Bible) pour voir comment il les désignerait. Deuxième lien entre l'animal et l'humain, celui du langage. Ils reçoivent chacun un nom, le leur, mais à aucun d'eux le 'ādàm ne peut dire: "mon vis-à-vis! ", "l'os de mes os, la chair de ma chair ». Ou comme l'Adam de Jean Grosjean: «Je ne te connaissais pas mais je te reconnais ${ }^{6}$."

Notre curiosité devra se résigner à ne rien entendre des noms imposés par la première créature humaine, à son premier usage de la parole, si elle ne s'en est pas tenue à une dénomination mentale. Nous reste du cortège une liste par genre et par espèce. Selon la variante retenue par la TOB de 1997, la catégorie "être vivant» $(2,19 b)$ reconnue chez tous englobe les espèces: bétail, oiseaux du ciel et bêtes des champs $(2,20)$. L'énumération est légèrement plus brève que la nomenclature-type de Gn 1,26: poissons de la mer, oiseaux du ciel, bêtes sauvages (= des champs), bestiaux, petites bêtes du sol' ${ }^{7}$. Elle témoigne cependant du même travail de systéma-

6. J. Grosjean, Adam et Ève, Paris, NRF, Gallimard, 1997, p. 29.

7. Littéralement: les bestioles qui rampent sur la terre. La traduction adoptée vise à inclure les animaux de petite taille et les insectes non rampants clairement compris dans la volonté d'exhaustivité du contexte. 
tisation derrière l'affabulation. Cet indice venu de l'énonciation mérite d'être pris en compte.

\subsection{Genèse 3}

La scène bucolique du rangement des animaux dans la nouveauté $\mathrm{du}$ monde ne constituera cependant pas le fin mot du rapport de l'humain et des bêtes. Car, c'est l'irruption d'un certain animal au jardin qui sera la pierre d'achoppement du cours de l'activité créatrice de Yahvé, jusque-là sans autre histoire que celle d'une suite d'essais et de progrès. Le serpent était une bête des champs $(3,1)$, dite aussi bête sauvage. Il n'appartenait donc pas au territoire privilégié aménagé par Yahvé, à l'ici construit par le texte comme opposé à un ailleurs indifférencié, issu également du Créateur mais poursuivant sa propre évolution parallèle que la Genèse ne se préoccupe pas de raconter. Verger d'arbres fruitiers et steppe semblent d'abord perméables un à l'autre. Après l'expulsion du couple humain, l'entrée du jardin sera bloquée par des chérubins, génies à forme mi-humaine mi-animale, armés du glaive de la foudre. À ce point du récit, le lecteur n'en soupçonnait même pas l'existence. Il retrouvera leur représentation encadrant l'arche dans la description de l'arche d'alliance du Temple de Salomon et du Temple post-exilique.

De toutes les bêtes des champs, le serpent était aussi la plus astucieuse, la plus rusée. Il était le plus 'arûm, joué par le texte avec deux humains incarnant, eux, l'autre face du même mot hébreu: des 'arummîm, c'est-à-dire des êtres nus, complètement vulnérables, sans apprêts au point de n'en avoir même pas conscience $(2,25)$.

L'animal prend la parole à son tour pour la première fois. Il reprend la première parole de Dieu en Gn 2 pour en donner sa lecture. Il s'adresse à la femme: "Élohîm vous a dit...». La version grecque de la Septante porte, de façon plus intellectuelle: "Pourquoi Dieu vous a-t-il dit? » Le serpent énonce le premier pourquoi adressé au Tout-Puissant, la première revendication à la compréhension venue de la création jusque-là au pôle de la réception totale. C'est d'ailleurs la réponse à ce pourquoi qu'il donnera à la femme dans la révélation d'une stratégie divine derrière la formulation de l'interdit 
de manger de l'arbre de la connaissance du bon et du mauvais: vos yeux s'ouvriraient et vous seriez comme des dieux, comme des élohîm. Yahvé entendait protéger ses prérogatives divines.

Il n'y a pas lieu de suivre ici les circonvolutions logiques d'un dialecticien à la hauteur de sa réputation d'habileté. Et pourtant la femme rétablit avec aisance la formulation exacte du précepte, faisant ainsi mentir les commentaires des Pères de l'Église d'un lointain futur sur sa rationalité déficiente, son incapacité à la réflexion, sa lascivité de femelle qui ouvre la porte à toute tentation. Néanmoins, l'homme, la femme, et le lecteur encore plus, perdront pied dans la torsade de demi-vérités, de demi-faussetés, de distorsions entre les dits du serpent, les dits de Yahvé-Dieu (Yahvé-Élohîm) antérieurs et subséquents à la transgression, les dits de l'énonciation qui ne cessent de correspondre ou de diverger du stade de l'interdiction à celui de la sentence, et cela en l'espace de quelques lignes.

Une logique narrative toute simple nous invite à relire, à la lumière des sentences des coupables, cette interdiction brouillée par le serpent. Or, cette rétro-lecture donne raison, contre Ève, à l'inquiétant Survenant: le couple mange, leurs yeux s'ouvrent, et, plus grave encore: Yahvé-Dieu dit: Voilà que l'humain est devenu comme l'un de nous pour la connaissance du bon et du mauvais $(3,22)$. À la différence de l'homme et de la femme, l'animal semble avoir lu Gn 1,26: «...à l'image de Dieu il les créa ${ }^{8}$ ». La rétro-lecture lui donne encore raison contre Dieu quand il déclare: «Pas du tout! Vous ne mourrez pas!» En effet, l'homme et la femme ne meurent pas le jour où ils ont mangé de l'arbre défendu, comme le leur avait annoncé ou les en avait menacé leur Créateur (2,27).

Entre les perplexités soulevées par l'herméneutique du serpent et celles de l'acception du mot mort pour ces êtres narratifs qui ne connaissent encore que la vie, les lecteurs friands d'énigmes pour-

8. Ce serpent-là admettrait la réponse par la différence des dates de rédaction de Gn $2-3$ et de Gn 1. Elle vaut pour Adam et Ève, mais comment y échappe-til ? Son intelligence subtile relancerait sans doute le problème méthodologique soulevé par l'adoption de la perspective de la lecture qui est la nôtre et, derrière elle, celui de la nature du corpus biblique. 
ront savourer longuement une des suites de la dégustation de l'arbre de la connaissance par leurs ancêtres. S'ils acceptent l'enjeu de ne pas introduire dans leurs réflexions la notion de mort spirituelle étrangère à la Bible, et de ne pas réduire trop vite l'épisode à la découverte de la sexualité génitale. Nudité, absence de honte, symbolisme du serpent, scène de séduction par la magie de l'arbre et du discours, réaction de pudeur de la confection de pagnes, tout y est. Une lecture freudienne des textes anciens est toujours possible et éclairante. À la condition de ne pas oublier non plus que d'autres symboles et symboliques sont partie intégrante de la matérialité de ces textes. Ainsi le serpent qui nous intéresse était-il dans l'antiquité porteur de valences positives que nous ne lui connaissons pas. Lié à la sagesse et à la gnose, il a déjà fait l'objet d'un culte en tant que bienfaiteur de l'humanité. Des sectes, dites Ophites (du grec ophis, serpent) répandaient ses enseignements. Qu'en était-il au viII ${ }^{\mathrm{e}}$ siècle A.C. chez les Hébreux de la cour d'Ézéchias?

Il nous suffira, pour notre propos, de jouer d'une lecture hors de l'axiologie démoniaque et sexuelle du tentateur pour voir toute la scène, et ses traductions, basculer dans un autre registre. À l'ouverture de leurs yeux, ce qui s'offre au regard de l'homme et de la femme, c'est leur nudité, leur dénuement, leur complète vulnérabilité. Le narrateur de 2,25 lie nudité à honte ${ }^{9}$ : " tous deux étaient nus et ils n'avaient pas honte l'un devant l'autre». La réponse de l'homme à Yahvé-Dieu en 3,10 relie nudité à peur: "J'ai entendu ton pas dans le jardin... j'ai eu peur parce que je suis nu...». Premier réflexe à cette peur, qui n'est pas la honte et la culpabilité mais l'angoisse de l'absence de protection: se couvrir. Il en résulte un revêtement élémentaire, un pagne de feuilles de figuier. Le végétal jusque-là garde-manger devient garde-robe. Il sera remplacé par des tuniques de peaux avant l'expulsion dans l'inconnu et l'inculte. L'animal devient source de protection pour l'humain qui naît sans

9. Le traducteur, lui, ignore si l'équivalent lexicographique qu'il emploie exprime l'acception du terme hébraïque, C'est le drame de la traduction des lexèmes de l'ordre de la passion, y compris à l'intérieur de la même langue à travers son histoire. 
plumes, sans écailles et sans fourrure. Il reste que c'est à un animal qu'a été dévolu le rôle de tester l'humain et la planification du Créateur, non à un ange, un démon, un élohîm ou un sphinx. Il n'a d'extérieur à la programmation divine que de surgir de l'extérieur du jardin.

La sentence ne se fait pas attendre. Parce que le serpent a trompé la femme et l'homme, il est maudit ('ārûr) entre tous les bestiaux et toutes les bêtes sauvages et réduit à l'état de rampant et à la poussière du sol comme misérable régime alimentaire. La texture même du vocabulaire hébraïque de Gn 3 porte sa trajectoire de déviance: le serpent passe de 'arûm à 'ârûr à travers sa rencontre avec deux 'arûmmîm. Yahvé-Dieu qu'il a défié l'enferme de surcroît dans une guerre de lignage sans merci, se perpétuant avec les générations, entre sa descendance et celle de la femme qui le vaincra, blessure à la tête contre morsure au talon. Première manifestation d'un lien entre le sort des animaux et celui des humains dans leur déchéance de l'harmonie originelle.

\section{Les animaux de l'Alliance - Gn 9,9-17}

Après le défilé des animaux devant Adam pour la cérémonie de leur dénomination, le temps fort par excellence de leur présence dans la Bible, c'est leur entrée dans l'arche de Noé et leur sortie au soleil d'un monde nouveau. De la reproduction de cette arche dans l'histoire de l'art et chez les marchands de jouets, on connaît généralement mieux la faune que la famille du patriarche, et sans doute que le motif de la solution extrême du déluge. La première génération des humains s'est sabordée elle-même. L'extinction de la branche d'Abel assassiné par son aîné et l'éviction de celle de Caïn, le meurtrier ostracisé, ont conduit à une seconde Genèse reprise dans les mêmes termes que la première ${ }^{10}$. Le livret de la descendance d'Adam s'ouvre maintenant par le nom de Seth, qui n'est plus présenté comme le substitut d'Abel $(4,25)$ mais comme un commencement

10. Comparer 5,1-3 à 1,27-28. 
absolu. Ce fils de la deuxième chance engendrera à son tour ${ }^{\circ} E n o ̂ s h$, nom qui signifie Homme au sens inclusif d'anthrôpos en grec. Mais les générations se succèdent et la dépravation et la violence couvrent la terre. Dieu vit que cela était mauvais, très mauvais.

Yahvé regretta alors amèrement la création de l'homme (6,6-7) s'en affligea dans son cœur et dit: "Je vais effacer de la surface du sol les hommes que j'ai créés, de l'homme jusqu'aux bestiaux, petites bêtes du sol et oiseaux du ciel. » La formulation de ces versets englobe sous le terme générique "homme» les autres catégories des vivants, comme si elles étaient son prolongement. L'animal est entraîné de soi dans le cataclysme. De la même manière qu'un juste et sa famille est épargné, un couple de chaque espèce animale sera aussi sauvé. Et Yahvé ne limite pas l'échantillonnage des bêtes au bétail et à la basse-cour de Noé. Ni non plus aux dimensions de l'arche; selon les vérifications d'Origène, elle «ne contiendrait même pas quatre éléphants» (seconde homélie sur la Genèse)! Cette constatation soulève la question du construit littéraire de l'épisode du déluge. Nous continuerons à l'aborder sous l'angle intrigant des nomenclatures d'animaux, à propos desquelles ces acteurs ont déjà commencé à prendre forme figurative et, peut-être, actantielle dans le récit biblique global ${ }^{11}$. Les rédacteurs les répètent avec la minutie de recenseurs officiels. Nous y suivrons les quelques variations apportées au modèle de base énoncé dans le projet de Dieu en 6,7: homme, bestiaux, petites bêtes du sol et oiseaux du ciel. Poissons et créatures marines semblent échapper au moyen d'extermination choisi. Le Tout-Puissant n'a pas à expliquer au lecteur qu'inondations et crues des eaux violentes bouleversent les fonds marins et compromettent

11. De Gn 6,5 à 9,17, nous en retrouvons treize occurrences, souvent accompagnées d'un verset-synthèse : la liste des êtres vivants ciblés par le verdict de Dieu (6,7.13); désignés comme occupants de l'arche $(6,17.18 b-20 ; 7,1-3)$; effectivement entrés dans l'arche (7,7-9.13-14.15-16); éradiqués par le déluge (7,21$23)$; sommés de sortir de l'arche (8,1.15-17); émergeant de leur refuge (8,18-19); destinataires d'un nouvel ordre (9,2-4); élus partenaires d'une alliance avec Dieu (9,9-10.12; 9,15-17). 
le degré de survie des poissons! Le mythe non plus n'a pas à justifier sa logique.

\subsection{Les instructions de départ}

La liste de 6,7 ne mentionnait pas les bêtes sauvages, 6,18b-20 la reprend en la précisant. C'est de tout être vivant, de toute chair (hébreu : bāśār) qu'il s'agit (v. 19) et c'est par couple, mâle et femelle de chacune des espèces, que Noé doit procéder au tri des futurs survivants. Quant au couple humain, il a droit à deux générations. Entreront dans l'arche "Noé, ses fils, sa femme et les femmes de ses fils ». Si 7,1-3 constitue un doublet immédiat, et dans sa proximité syntagmatique et dans son cadre de base, ces versets apportent un ajout inattendu, soit l'existence d'une nouvelle distinction inter et trans-catégorielle chez les animaux. Certains sont purs, d'autres impurs. Parmi le bétail (hébreu: běhēmāh, běhēmôt au pluriel), Noé prélèvera sept couples des animaux purs, un couple seulement des impurs mais sept couples des oiseaux du ciel. Le tressage des deux traditions repérées en Gn 1 et Gn 2 (voir note 4) explique le doublet. Curieusement, cependant, c'est la tradition yahviste qui se préoccupe de la pureté des animaux et non la sacerdotale qui la théorisera et «théologisera » au livre du Lévitique. Plus curieusement encore, Dieu, dans son opération de purification radicale au prix d'hécatombes de chair humaine et animale, assure la perpétuation des animaux dits impurs. Ce fait troublant soulève toute la question de l'acception de la pureté/impureté en jeu.

\subsection{L'embarquement}

Un second doublet répète le premier, marqué par le défilé des animaux venant à Noé dans l'arche pour leur salut et rappelant leur procession devant Adam pour leur dénomination. Le premier volet, apport de tradition yahviste, continue de distinguer les bestiaux purs et impurs (7,7-9). Le second, de tradition sacerdotale, fournit la liste la plus longue incluant les bêtes sauvages et la classification par espèces et par couples de toute créature ayant souffle de vie, un mâle 
et une femelle de toute chair (7,13-16), hors de la répartition entre purs et impurs. «Et Yahvé referma la porte sur Noé.»

\subsection{Le déluge}

Les grandes eaux de l'abîme séparées et harnachées lors de la création furent réunies, et ouvertes les écluses du ciel. Une troisième reprise jumelée de 6, 7 sert à enregistrer les pertes du déluge. La liste familière est coiffée dans les deux cas d'une constatation globale qui surclasse tout pathos. "Avec la crue des eaux qui recouvrirent les montagnes expira toute chair qui remuait sur terre...»(7,20-21). "Tout ce qui avait une haleine de vie dans les narines, c'est-à-dire tout ce qui était sur la terre ferme, mourut.» $(7,22)$ Le verset 23 boucle la boucle: "Ainsi le Seigneur effaça tous les êtres de la surface du sol», tous ceux que 6,7 avait nommés.

\subsection{Le débarquement}

Long atterrissage à la verticale par assèchement des eaux qui couvraient les sommets des plus hautes montagnes plutôt qu'arrivée à un port, le débarquement tient narrativement dans la double reprise de la liste complète, titrée: "Dieu dit à Noé: Sors de l'arche, toi et...» $(8,16)$ et «Noé sortit et avec lui...» $(8,18)$. Bêtes sauvages et bestiaux apparaissent et disparaissent à tour de rôle, mais assimilés dans les deux cas à l'intérieur de "toutes les bêtes (hébreu: tous les vivants) qui sont avec toi ». Troisième défilé encyclopédique de l'espèce animale. Pour se transformer en renaissance, la fin du monde va emprunter les termes du récit de la première création. Si les préceptes de fécondité, de prolifération seront les mêmes, les relations entre le monde humain et le monde animal subiront une transformation tragique et inattendue. Le texte en présente laconiquement le comment mais non le pourquoi.

\subsection{Les animaux de l'Alliance}

Le rédacteur yahviste devance le fil de l'histoire et prête à Noé mettant pied à terre un geste qui relève de l'alliance mosaïque. On 
ignore sous quelle forme Abel avait offert à Yahvé des premiers-nés de son petit bétail, mais Noé, lui, procède selon des règles combien postérieures, construisant un autel, prélevant de tout bétail pur et de tout oiseau pur des victimes qu'il immole en holocauste. Jusqu'en l'an 70 de notre ère, jusqu'à la destruction du Temple de Jérusalem, les animaux seront désormais voués à alimenter de leur vie l'institution sacrificielle.

La fumée de l'holocauste s'avère efficace. Pacifié, le Dieu du déluge bénit Noé et ses fils avec les mots du "sixième jour ", mais qui visent maintenant un monde resté à jamais marqué par le cœur mauvais de la race humaine. La reprise de la liste totalisante (9,2-3) aboutit à montrer toutes les catégories de vivants enfermées sous la crainte et l'effroi suscités par les humains. Tout ce qui remue et vit sur terre, dans la mer et dans le ciel est «livré entre vos mains ». Tout ce qui remue et vit vous servira désormais de nourriture, comme auparavant le végétal. De végétariens qu'ils étaient, humains et animaux deviennent carnivores et carnassiers, et proie les uns des autres. "Toutefois vous ne mangerez pas la chair avec sa vie, c'està-dire son sang. De même de votre sang, qui est votre propre vie, je demanderai compte à toute bête et j'en demanderai compte à l'homme. » $(9,4-5)$ La restriction de la première phrase de la citation traversera tout l'Ancien Testament et pèsera d'un grand poids dans l'expansion du christianisme naissant.

Sur le mont Ararat, la gent animale apprend qu'un lourd servage l'attend. Ses maîtres n'héritent pas d'un destin plus glorieux: être l'effroi et l'épouvante des autres vivants de la planète. En revanche, un arc-en-ciel se déploie avec bonheur au-dessus d'un récit qu'il faut se garder d'exorciser trop vite de son horreur, sous peine de n'en rien retirer. Enfin, «Dieu dit à Noé et à ses fils. Je vais établir mon alliance avec vous, avec votre descendance après vous et avec tous les êtres vivants qui sont avec vous...» (9,8-19). Suit la liste familière élevant ici la totalité des animaux au rang de partenaires de l'Alliance, "même les bêtes sauvages" précisent les rédacteurs ${ }^{12}$. La

12. Voir également Osée 2,20 et Joël 1,18-20. 
lectrice se surprend à regretter que ni Madame Noé ni ses bellesfilles ne soient nommées au moment solennel de la ratification d'une alliance perpétuelle entre Dieu et «toute chair qui est sur la terre ", pour toutes les générations futures ${ }^{13}$. Elles l'étaient pourtant à l'heure de l'embarquement pour la grande épreuve et elles sont recensées à celle du débarquement $(8,8)$. Or, «Dieu dit à Noé et à ses fils...»

Le terrible épisode du Déluge se referme donc - et ouvre la suite - sur une Alliance. D'abord annoncée par Dieu à la construction de l'arche comme un pacte «avec toi » Noé, elle est étendue le moment venu à tout être vivant choisi pour survivre. La gent animale y gagne un statut nouveau. D'une part, les animaux pourront être dits à juste titre les animaux de l'Alliance, y compris les bêtes sauvages qui n'habitent pas avec les humains. Tous, ils portent le serment divin de ne plus jamais être exterminés par un déluge «tant que la terre durera » $(8,22)$. D'autre part, déjà soumis aux deux `ādām mâle et femelle lors de la création initiale $(1,28)$, ils deviennent nourriture des humains et matière de leurs offrandes sacrificielles. Désormais, ils sont donnés, ils sont livrés entre leurs mains $(9,2)$.

De Gn 6 à 9, la mise en discours des animaux s'est enrichie de traits additionnels. La nomenclature si souvent répétée par le texte de «bêtes sauvages, bestiaux, petites bêtes $d u$ sol, oiseaux du ciel,

13. Voir T. Findley, Not Wanted on the Voyage, Toronto, Viking, 1984. Timothy Findley mérite notre gratitude pour l'enchantement de ce livre écrit du point de vue de $\mathrm{M}^{\mathrm{me}}$ Noé et de sa chatte Mottyl et dédié «Against Despair» aux animaux qui ont partagé la suite de ses jours. L'arche y devient la maquette flottante d'une critique de la religion, des sociétés et des idéologies. Acidulée sinon acide, iconoclaste parfois, elle demeure à toutes les pages une fable hautement poétique et imprégnée d'une immense compassion. De la même encre, le premier chapitre de A History of the World in 10 Chapters de J. BARNEs, New York-Toronto, Alfred A. Knopf, 1989, p. 3-29, "The Stowaway», ou les mémoires d'un rat-de-cale, qui est un termite, "piratant» son passage dans l'arche, qui n'a rien d'un paquebot de croisière et où " only the penguins wore tailcoats» (p. 3). Dans la lignée de la réception du récit de Déluge, deux exemples fascinants d'une troisième position d'interprétation entre l'utilisation du texte biblique et les études critiques, soit celle de l'œuvre de création littéraire. 
créatures marines» est maintenant traversée dans chacun de ses groupes par une catégorie nouvelle: le pur/impur, appliquée jusqu'ici qu'aux animaux. Entrés dans l'arche soumis à l'humain dans la hiérarchie des vivants, ils en sortent livrés entre ses mains. Leur désignation spécifique comme détenteurs de souffle de vie s'étend à leur possession du sang de la vie, également tabou chez eux comme chez l'humain, devenus nourriture sur la table de l'homme et parfum d'agréable odeur sur l'autel de Dieu, ils vivront, et mourront, d'un rapport tout autre dans les deux cas. Pour les animaux de l'arche regagnant leurs habitats naturels sous l'arc-en-ciel de l'Alliance - et pour les lecteurs - l'impact de ces éléments nouveaux demeure encore à découvrir.

\section{Nourriture de Dieu et des hommes - Le Lévitique}

Nous supposons que nous lecteurs sérieux avons lu, dans l'ordre, l'Exode après la Genèse et abordons du même mouvement le troisième livre du Pentateuque, le Lévitique. Rares sont les ouvrages qui commencent par la conjonction «et», qui les relie directement à un livre antérieur et dont la contraction avec le premier mot du texte sert de titre, comme dans la Bible hébraïque: «Et-il-appela ${ }^{14}$ ». La littérature rabbinique, elle, le désigne par son contenu «la loi ou l'enseignement des prêtres " et le nom de Lévitique, où on reconnaît celui du lévite, membre de la tribu sacerdotale de Lévi, provient de la Septante. Nous y entrons par le sentier des animaux ouvert après le déluge, c'est-à-dire celui des deux isotopies sacrificielle et alimentaire et de leurs parcours narratifs et figuratifs ${ }^{15}$.

14. Littéralement: Et-il-appela Moïse et-lui-parla Yahvé de la tente de la rencontre et (lui ) dit. (Selon les traductions: tente de la réunion, de la rencontre (TOB), du rendez-vous $(\mathrm{BJ})$, du témoignage (BA ou Bible d'Alexandrie, traduite du grec de la Septante), d'assignation (La Bible. Nouvelle traduction).

15. Cette première incursion exploratoire s'en tiendra à la cohérence propre au Lévitique, sans ouvrir la discussion des passages parallèles, et parfois divergents, du Deutéronome. 


\subsection{Nourriture de Dieu: l'animal sacrificiel}

Si quelqu'un veut offrir un présent à Yahvé, c'est un animal qu'il lui apporte à la tente de la rencontre, lieu de sa présence (Lv 1,2). Il y sera transformé en «mets consumé» sur le feu de l'autel, par les prêtres investis par la consécration nécessaire de la dangereuse fonction de la manipulation du sacré. Dans un style législatif en si/alors, les sept premiers chapitres codifient les rites des différents types de sacrifice ${ }^{16}$. À travers la récurrence constante d'expressions «consacrées » par l'usage, on déduit très vite que sacrifier signifie "présenter à Yahvé un mets consumé " en tout ou en parties (à titre d'exemple, Lv 23,36-37); que sacrifice veut dire nourriture de Dieu sublimée en parfum agréable et apaisant pour lui. L'animal entier, vidé de son sang, monte en fumée vers Dieu dans le cas de l'holocauste, et en parties choisies seulement pour les autres sacrifices, y compris dans le seul sacrifice non sanglant, celui de l'offrande végétale et de la cérémonie apparentée de l'offrande des prémices des récoltes.

L'ensemble de ces prescriptions sacrificielles compose un menu de chairs et de graisses animales, de pains, de galettes et de gâteaux cuits au four, sur une plaque ou à la poêle, nourriture dite sainte, humée par Dieu, ingérée par les prêtres et leurs maisons dans les parts qui leur sont dévolues par la loi de leur état, redistribuée à l'offrant et à la famille selon une répartition rigoureuse et des circonstances bien précises.

En structures narratives, l'animal sacrifié occupe le centre d'une chorégraphie minutieuse exécutée par l'offrant et les prêtres. Plus que son rôle actantiel nous intéressent ici les traits figuratifs à travers lesquels l'énonciation non seulement décrit l'animal-victime mais le construit. Ils se répètent en nombre fini des espèces admissibles et toujours les mêmes, d'une section à l'autre du Lévitique; on les retrouve dans le rituel de base des sacrifices, à l'investiture des

16. Pour une brève présentation particulièrement claire et compétente du système sacrificiel de l'Israël biblique, voir: G. Couturier, "Le sacrifice d'“action de grâces” ", Église et Théologie, 13 (1982) p. 5-34. 
prêtres, au rituel des grandes fêtes de l'année liturgique, enfin dans les sacrifices de purification dans la vie courante de la communauté, des couples et des individus. Seul le nombre d'animaux immolés à l'intérieur d'une même catégorie va varier selon les types de sacrifices. Au sujet de l'holocauste, 22, 19-20 condense commodément les traits de la victime autorisée, mais leur relevé dans l'ordre syntagmatique de la lecture apporte des précisions importantes.

L'animal du sacrifice sera prélevé parmi le gros bétail (bovidé), le petit bétail (ovidé) ou, en cas d'indigence de l'offrant, chez les pigeons et les tourterelles. On devra choisir une bête mâle. Elle devra être sans défaut et sans tache. Provenance, sexe et intégrité physique circonscrivent les conditions d'acceptabilité de la victime par Dieu. Une fois agréé et engagé dans le processus sacrificiel, l'animal acquiert un quatrième trait ou figure: "c'est une chose très sainte » (6,17-22). Il se mange dans un endroit saint, par le prêtre qui a présidé au sacrifice ou "par tout mâle qui fait partie des prêtres ». Tout ce qui touche à sa chair est sanctifié et le récipient d'argile où il a cuit doit être brisé, le récipient de bronze récuré et rincé à l'eau (v. 19-21). Ces affirmations parmi d'autres sont extraites intentionnellement d'un passage sur le sacrifice pour le péché; elles ont l'avantage de montrer que l'animal sacrifié n'est ni chargé de péchés, ni maudit, ni châtié à la place de l'offrant mais haussé à la dignité de don pour Dieu ${ }^{17}$.

La description du déroulement des divers sacrifices fait émerger des traits supplémentaires moins clairement énoncés mais omniprésents dans le texte. Quant à la provenance de la victime - Elle est tirée des animaux domestiques et non des bêtes sauvages et des produits de la chasse. Elle appartient donc aux animaux qui partagent la vie du peuple de l'Alliance. Qu'elle soit de gros, de petit bétail ou de la population d'oiseaux familiers (seuls pigeons et tour-

17. Il n'existe qu'une seule occurrence du rite du bouc émissaire connu dans tant de civilisations antiques et modernes. Au Yom Kippour, le grand-prêtre charge de tous les péchés d'Israël l'un des deux boucs présentés pour le sacrifice d'expiation et le fait expulser au désert. Ce bouc dit «pour Azazel », le démon des espaces désertiques, ne sera surtout pas offert à Yahvé (16,7-10.21-22). 
terelles sont nommés) est déterminé par l'importance du sacrifice auquel elle est destinée. La gradation parmi les sacrifices en crée une chez les animaux ${ }^{18}$. Quant au sexe de l'animal — Il n'est jamais précisé pour les oiseaux offerts par les indigents ou prescrits pour les sacrifices de purification courants. Cependant, la distinction sexuelle est toujours mentionnée et strictement imposée pour l'holocauste, le sacrifice de communion ou de paix, le sacrifice pour le péché (batțāat), celui dit de réparation ( $(\bar{a} s \bar{h} \bar{a} m$ ) et pour la combinaison de différents sacrifices exigée pour le Yom Kippour, l'investiture des prêtres et les premiers sacrifices accomplis par eux. Le sacrifice par excellence, l'holocauste, n'admet qu'une victime mâle, de même que les sacrifices de consécration des prêtres et de leur entrée en fonction, et le rituel du Jour du Grand Pardon.

Dans les sacrifices pour le péché et la réparation, c'est le statut social du pécheur qui détermine le sexe prescrit. Pour les péchés du grand-prêtre, de la communauté d'Israël entière, des chefs, une victime mâle est de règle; pour les péchés d'un homme du peuple et les sacrifices de réparation d'un individu, une victime femelle, chèvre ou brebis. Enfin, une agnelle suffit pour le sacrifice pour le péché d'un lépreux à sa guérison, sacrifice couplé à un sacrifice de réparation et à un holocauste qui requièrent un agneau chacun. Dans l'ensemble du Lévitique, la hiérarchisation du mâle et de la femelle a pour écho, et réciproquement, celle de l'homme et de la femme dans leur rapport aux espaces sacrés et dans leur accès à la fonction de sanctification. Malgré l'ouverture de quelques brèches timides, cette législation de l'époque du Second Temple (537 A.C.) perdure encore dans certaines branches du judaïsme et du christianisme.

Quant à l'intégrité physique - Le texte pose une autre correspondance entre victime et sacrificateur. À victime mâle (dans la majorité des cas), parfaite selon les canons de son espèce, prêtre

18. La dimension économique de ces choix chez un peuple d'éleveurs ne pourra être abordée ici. Nous nous en tiendrons à celle qui paraît à la surface de la manifestation du texte, soit la dimension théologique énoncée plus loin comme résolution des ramifications de la structure sacrificielle complexe mise en place. 
également mâle, anatomiquement sans défaut. Tout «mâle» $(6$, $11.22 ; 7,6)$ normalement constitué et qui fait partie des fils d'Aaron est prêtre d'office. Celui qui est handicapé, difforme ou souffrant d'une malformation génitale «ne peut présenter la nourriture de son Dieu » (21,16-23). À titre de membre d'une famille sacerdotale, il est cependant autorisé à recevoir sa part de ce qui est dévolu aux prêtres dans la viande sacrifiée. Le code réglemente même le sort de la fille du prêtre. En quelles circonstances peut-elle ou ne peut-elle pas partager «la nourriture de son père » $(22,12-13)$, lui qui est consacré à «la nourriture de son Dieu»?

Fort curieusement, au fil des dix premiers chapitres du Lévitique sur sacrifices et sacerdoce, nous n'avons pas rencontré le trait figuratif du pur/impur appliqué à l'animal sacrificiel ou destiné à être offert à Yahvé. Il est apparu dans des phrases secondaires, mais à propos d'impuretés involontaires et inconscientes contractées par des humains au contact de cadavres d'animaux domestiques, de bêtes sauvages ou de bestioles, à propos d'impuretés chez les humains donc (5,2-3). Et de l'impureté humaine, de son sens, ces versets ne nous laissent que cette définition déroutante: "toute impureté qui rend impur»!

Plus curieusement encore, c'est au sujet de l'animal comme aliment des humains que le législateur sacerdotal va déployer la dimension du pur/impur, la réintégrer dans le sacrificiel, puis conduire cette "Loi de la pureté » à son enracinement et sommet théologique dans ladite «Loi de sainteté » des chapitres 17-26.

\subsection{Nourriture des hommes}

Que deviennent les animaux de l'Alliance noachique, puis mosaïque, sur ce deuxième sentier tracé pour eux dans le corpus biblique, soit l'isotopie alimentaire? La section des Règles relatives au pur et à l'impur y répond on ne peut plus clairement en son chapitre d'ouverture (Lv 11,1-47). Écrit sur le mode de la prescription et de l'interdiction, il ne laisse aucune place à la facultativité, au choix ou au refus de ce système diététique. Ses versets de conclusion auraient pu lui servir d'en-tête: v. 46: «Telle est la loi concernant les animaux 
(litt. les bestiaux), les oiseaux, tout être vivant qui se meut dans l'eau et tous les êtres qui pullulent sur terre. 47: Elle a pour but de séparer le pur et l'impur, les bêtes que l'on peut manger et celles que l'on ne doit pas manger.»

On reconnaît la nomenclature de base de Gn 1,26, les bêtes sauvages en moins; ses éléments servent de démarcation aux différents paragraphes de la séquence globale et s'ouvrent chacun en un éventail de bêtes connues et inconnues, avec leurs noms, leurs particularités anatomiques et motrices. Contre nos attentes, les versets 1 à 44 ne s'appuient pas sur l'énoncé d'une définition du pur/impur, pour ensuite en déduire le catalogue des animaux admis à la consommation. À l'inverse du v. 47, ils statuent sur la liste de ceux qu'on peut manger ou qu'on doit ne pas manger, pour en conclure qu'ils sont purs ou impurs. Sauf quand ils introduisent un nouveau trait figuratif de l'ordre du dégoût et de la nausée qu'on n'arrivera à rendre, à mon avis, qu'à travers un meilleur éclairage des contextes grâce à des analyses littéraires qui tardent encore. On trouve en traduction tantôt: abominations, horreurs, immondes (BJ), tantôt: animaux à fuir et à éviter, ou interdits (TOB), ce qui a pour effet ou de noircir le texte biblique ou de l'adoucir. Quand ce terme apparaît, on retrouve l'ordre causal du v. 47 de l'abomination à l'interdiction.

1-8 - Parmi les animaux terrestres on pourra manger tout animal qui possède des sabots, mais des sabots bifides, et qui rumine. Ceux à qui il manque un de ces trois critères de sélection, "vous les tiendrez pour impurs» (BJ), "pour vous ils sont impurs» (TOB). Sont distingués, avec exemples, les cas de l'animal sans sabot ou plantigrade (27. 29), à sabot non fendu (26) ou qui ne rumine pas. Notoire jusqu'à nos jours, l'impureté du porc repose sur le fait que se déplaçant sur des sabots et des sabots bifides, il ne rumine cependant pas.

9-12 - Parmi les animaux aquatiques, on pourra manger tout ce qui porte écailles et nageoires et vit dans l'eau des mers ou des fleuves. Mais tout ce qui vit dans l'eau sans répondre aux deux premiers critères des écailles et des nageoires, "vous les tiendrez pour 
immonde » et «vous aurez en dégoût leurs cadavres » $(\mathrm{BJ})^{19}$. Le texte s'en tient ici à la déclaration de principe, sans fournir d'exemples de la faune marine remontée dans les filets de pêche.

13-19 - Quant aux oiseaux, les critères de sélection ne sont pas donnés. La simple déclaration que certains sont immondes, interdits et abomination précède le commandement de s'en abstenir qui en découle. Suit une longue liste des oiseaux proscrits, du vautour à la chauve-souris en passant par l'autruche, la cigogne et l'ibis.

20-23 - Toutes les petites bêtes ailées qui marchent sur quatre pattes, différentes en cela des volatiles bipèdes, on les tiendra pour immondes, donc on ne pourra en manger. Sont admises à la consommation, celles qui ont, articulées à leurs pattes, l'équivalent d'une jambe qui leur permet de sauter, comme les différentes espèces de sauterelles.

24-40 - Toujours au sujet des animaux immondes ou abominables, le texte reprend maintenant le vocabulaire du pur/impur pour expliquer le danger qu'ils représentent. Leurs contacts avec l'humain l'insèrent dans un réseau de contamination. Il se propage des personnes aux vêtements, aux objets, aux ustensiles et aux graines de semence, et on ne peut s'en libérer que par des rites de purification, car ce qu'il propage, c'est leur impureté. De cette interruption dans l'énumération zoologique ressort le caractère englobant et régulateur de la catégorie du pur/impur par rapport aux traits figuratifs de l'abomination et de l'interdit.

29-30; 41-44 - Reste le cas des petites bêtes qui pullulent sur la terre ferme, rampantes ou à quatre pattes ou plus. Elles sont toutes immondes et on n'en mangera pas. Le verset 29 admet des excep-

19. La TOB traduit: vous sont interdits... et vous mettez l'interdit sur leur cadavre. En 1988, la Bible d'Alexandrie rend le grec de la Septante, lui-même traduction de l'hébreu du texte massorétique, par: ils seront abomination pour vous...et vous aurez en abomination leurs cadavres. Une telle distance entre les options laisse soupçonner une zone d'incertitude non résolue. Immondes, interdits et abominations ne sont guère des équivalents sémantiques. 
tions, puisque celles «qui pour vous sont impures sont la taupe, le rat et les lézards ", ce qui laisse aux consommateurs la majeure partie d'une immense population. Ici se termine l'effort colossal d'une classification du monde animal poussée à l'obsession...ou bien d'un subtil jeu de l'esprit à partir de critères les plus simplistes, pattes, nageoires et ailes. La célèbre anthropologue Mary Douglas a voulu vérifier dans une incursion au royaume biblique depuis longtemps souhaitée ${ }^{20}$. Elle arrive au résultat qu'effectivement la grille sacerdotale basée sur les moyens de locomotion peut ranger tous les animaux connus. Voir l'éloquence des tableaux soumis aux pages 139 et 156.

La conclusion du chapitre 11 enracine dans un raisonnement hautement théologique les prescriptions alimentaires les plus quotidiennes tirées d'observations fauniques les plus concrètes. "Ne vous rendez donc pas impurs avec toutes ces bestioles qui rampent sur la terre $[\ldots]$ vous vous êtes sanctifiés et êtes devenus saints, car je suis saint [...] vous serez donc saints parce que je suis saint» (44-45). Cette fin de séquence oppose la figure impurs à celle de saints et non plus à celle de purs selon l'articulation logique de la langue; elle relance surtout la question latente du sens de pur/impur. Le dictionnaire des langues de départ et d'arrivée de la traduction n'y répondra pas de façon satisfaisante, comme en témoignent les tentatives de rajustements dans les notes des bibles récentes. À défaut de pouvoir développer l'analyse de discours qui éclairerait la véritable charge sémantique des notions en jeu, je m'appuierai sur une étude déjà réalisée à propos des taux différents d'impureté de l'homme et de la femme dans leur vie cultuelle et sociale, d'après les chapitres suivants du Lévitique ${ }^{21}$.

20. M. Douglas, Leviticus as Literature, Oxford, Oxford University Press, 1999. L'auteure y pratique une lecture par analogie qui tire le plan toujours controversé du Lévitique d'une correspondance avec les trois parties de la tente de la Rencontre, de la montagne du Sinaï et de la disposition du corps dépecé de l'animal sur l'autel.

21. O. Genest, «Mythical Roots of Women's Impurity in the Law of Leviticus: Gendered Mathematics of the Pure and Impure ", Canadian Journal of Law and Society/Revue Canadienne Droit et Société, 14/1 (1999) p. 9-30. 


\subsection{Purlimpurlabomination.}

Dans sa classification des êtres vivants (tableau 8.3)22, Mary Douglas subdivise les animaux terrestres en purs/impurs, les aquatiques et aériens en commun/abominable. Abominable est ainsi caractérisé par opposition à commun et traduit par "à éviter, à fuir ": "the rule is not to hate but to avoid or shun, and the do-not-touch rule protects the listed creatures ${ }^{23}$ ». En accord avec ces nombreuses déclarations où Dieu exprime sa sollicitude pour toute sa création, cette traduction n'en désamorce pas moins un texte qui se veut précisément emphatique au sujet du pur/impur. Or, le lexème abomination est bien attesté, avec la prescription de "abominer ", où le $a b$ latin rejoint le à fuir. À titre d'hypothèse, je le maintiens pour le moment dans toute sa brutalité, comme un intensif, un non-désir catégorique commandé au destinataire de la législation, et non comme une propriété monstrueuse de tel ou tel animal.

Le pur/impur apparaît d'abord comme principe de classification, du type du permis/défendu, avec ses applications et abstentions, ses sanctions et ses mesures de corrections, soit les rites de purification. Et la définition minimale du pur comme l'absence de l'impur. Et l'acception de l'impur, alors? On se souvient que des animaux dits impurs ont été désignés comme passagers de l'arche de Noé le juste. Pourtant l'occasion était belle d'effacer avec eux cette bavure de la création ou de réparer ces moments d'inattention d'un créateur débordé par sa créativité! Nous savons déjà — une simple lecture attentive nous l'apprend quand nous arrivons à la décanter de conceptions sacrificielles stéréotypées, autres que celle du Lévitique nous savons que l'impureté à éviter n'est pas de l'ordre moral mais cultuel, que le système sacrificiel de l'Israël biblique a pour intention première non la faute morale mais les manquements inconscients ${ }^{24}$

22. Douglas, Leviticus as Literature, p. 156

23. Douglas, Leviticus as Literature, p. 157.

24. Voir les chapitres 4 et 5 sur le sacrifice pour le péché. À l'introduction de chaque cas répertorié, le texte reprend sans se lasser: "quand on pèche par mégarde...", "si sans s'en rendre compte on touche n'importe quoi d'impur...». 
à l'impeccabilité cultuelle et que l'exigence de cette pureté irradie de la tente de la rencontre vers la vie sociale et intime du peuple de l'Alliance.

Nous savons que cette impureté-là n'est pas non plus de l'ordre esthétique, ni hygiénique (c'est la lecture d'Hitler dans son Mein Kampf), ni utilitaire (chameaux et chevaux protégés de l'autel fatal par l'impureté de leurs sabots), ni économique (les victimes sacrificielles étant prélevées du cheptel essentiel à la survie des familles), ni relatif aux animaux ailleurs destinés aux cultes idolâtriques, ni non plus de l'ukase arbitraire puisque le texte explicite ses raisons. Ces savoirs négatifs ne font que nous mener au seuil de la question. Le seuil de la réponse réside dans l'information syntaxique et sémantique que les multiples articulations avec le contexte du chapitre 11 injectent de facto dans l'acception du pur/impur.

Sémiotiquement, au niveau de surface, les formes figuratives du pur/impur se résolvent dans le propre-à/impropre-à et, en structure profonde, dans la catégorie sémique du même/autre. Les ponctions pratiquées par l'analyse sur de petites ou de vastes unités discursives aboutissent aux mêmes constatations. D'une part, les labyrinthes déroutants d'un catalogue de prescriptions et d'interdictions sans logique apparente, surpeuplés de tant d'acteurs en tant de situations disparates et pourtant concrètes et factuelles (à la différence de l'hyper-métaphorisation du discours gnostique, par exemple); d'autre part, une cohérence remarquable dans les moindres détails des passages déjà abordés, une fois que le lecteur a saisi l'organisation d'ensemble. Et si tel espace de texte résiste encore à la compréhension, heureux-se exégète ainsi préservé-e du leurre de la description sémiotique exhaustive, heureux texte sauvé de la mort par interprétation totalitaire à sens unique.

À un pôle de la catégorie de base, le même de l'ipséité et de la similarité qui crée le pôle opposé de l'autre, et réciproquement. Est dite pure la coïncidence parfaite avec soi-même, les caractéristiques de son espèce et son milieu écologique et social, selon les critères statués par la caste sacerdotale. Si on est un poisson digne de ce nom, con-forme à la morphologie de son espèce et à son milieu, on 
vit dans l'eau, on possède écailles et nageoires et on nage. On est alors une "pure" créature marine. Si on marche au fond de l'eau, comme les homards et les crabes, on ne correspond pas à la pureté de l'espèce, donc on est impropre à l'assimilation par des humains vivant en régime de pureté. Est déclarée pure également la similarité à l'intérieur des groupes, des classes, des réseaux d'appartenance. Ainsi: "n'accouple pas deux espèces différentes» $(19,19)$. De la même encre, la bestialité, c'est-à-dire les relations sexuelles entre humains et animaux est proscrite.

Un raffinement de la construction juridique et littéraire produit de plus des combinaisons croisées du même et de l'autre. Le code des relations sexuelles bannit le même de la famille, de l'inceste, et celui de l'homosexualité. Toute femme qu'elle est, la fille d'un prêtre peut consommer des mets de l'autel réservés aux prêtres en tant que "même» de son père. Par le mariage, elle devient autre et perd ce droit, mais le recouvre si, par veuvage ou divorce, elle revient à la maison de son père. Enfin, parmi les points encore obscurs, un cas de redoublement du même requerrait une analyse à la fois plus large et plus fine. Pourquoi la femme accouchant d'une fille est-elle deux fois plus longtemps impure (deux semaines +60 jours de purification de son sang) qu'après l'accouchement d'un garçon (1 semaine + 33 jours), la perte de sang étant la même ou, si elle diffère, ne relevant pas de la détermination sexuelle du bébé? Le redoublement du caractère mâle chez le prêtre et la victime sacrificielle appartiendrait-il à la même raison obscure? Et toute la question du poids du genre dans la tarification partiale du degré d'impureté de l'homme et de la femme, du mâle et de la femelle chez les animaux, établie par des prêtres mâles?

\subsection{Saint / impur}

Le plan théologique nous donne également sous le revêtement de la cohérence du même/autre, le pourquoi de ce comment du texte. Sa mise en discours transforme au fil des contextes, l'opposition purs/ impurs en saints/impurs. Dans les deux oppositions, l'impur demeure la cause du brouillage du lien entre Dieu et les humains; la purifi- 
cation par le sacrificiel restaure l'harmonie compromise. Mais le byzantinisme de l'observance - et sa grandeur - servis jusqu'ici à la lecture à travers le lexique de la pureté, vont trouver leur justification, non dans la recherche de l'humaine perfection ou l'obsession du juste pour sa propre pureté ou la hantise de déchoir d'une alliance incomparable, mais dans le lexique de la sainteté. "Vous vous sanctifierez donc pour être saints, car je suis saint " $(11,44)$ et c'est moi le Seigneur qui vous sanctifie» $(22,32)$.

Les contextes construits par l'énonciateur ont inoculé un investissement sémantique autre dans le lexique de la pureté. Non seulement la charge sémantique de pur a-t-elle varié, mais une nouvelle thématique a été mise en place, celle de la sanctification, avec ses rôles de sanctifiant et de sanctifié; elle surclasse nettement la thématique de la purification et ses rôles de purifiant et de purifié. Si on continue à se laisser guider par la matérialité du texte, saint s'oppose ici à commun, et non à profane qui appartient à la langue et à la rationalité des commentateurs. La définition réciproque de ces deux pôles contraires fait apparaître dans la sainteté l'élément de séparation et de consécration.

L'imposante architectonique du Lévitique se résorbe en une injonction radicale: vous établirez des distinctions parmi les réalités de votre monde; vous serez séparés et consacrés parce que je vous ai mis à part et réservés pour moi parmi les nations, moi qui suis séparé, le Tout-Autre. À un Dieu saint ( $q \bar{a} d \hat{o} s h)$, un peuple saint $(q \bar{a} d \hat{o} s h)^{25}$. Dans cette séparation, l'animal intégré au peuple de l'Alliance est lié, dans son souffle et dans son sang, au mécanisme de purification et de louange constante. L'analyse narrative le retrouve au poste actantiel de l'Adjuvant et non à celui de sujet d'état, ce qui, malheureusement pour les amateurs d'animaux, ne leur permet pas de parler de sanctification de l'animal dans l'Ancien Testament, malgré la consécration de certaines de ses parties sur l'autel.

25. Lv $11,44-45 ; 19,2 ; 20,7.26 ; 21,6-8$. 
Plus largement encore, sacré s'oppose à chaos. Le sacré a pour fonction de gagner sur le chaos et de le tenir en respect, jusque dans les relations avec la population animale. Dans la paix de l'Alliance «vous dormirez sans que rien ne vous effraie, je ferai disparaître du pays les bêtes malfaisantes ", mais «si vous vous opposez à moi [...] je lâcherai contre vous les bêtes sauvages qui vous raviront vos enfants, anéantiront votre bétail et qui vous décimeront au point que vos chemins deviendront déserts. » $(26,6.22)$

Sauvage ou domestique, du dehors ou du dedans, l'animal garde sa dignité de vivant. La loi du talion le couple à l'humain. L'homme qui frappe à mort un être humain est mis à mort; l'homme qui frappe à mort un animal le remplacera — «vie pour vie» (24,1718). L'hébreu emploie běhēmāh pour animal, donc la législation s'applique au bétail et on peut lire le précepte du point de vue du propriétaire lésé, sur l'isotopie économique. La justice-dette ne s'exerce pas de la même manière pour dommages causés aux humains ou aux animaux, la peine de mort ou l'infirmité identique infligées dans un cas, la compensation dans l'autre. Cependant, les trois derniers mots à la fin des versets 17 et 18, rédigés en parallélisme, nefesh pour nefesh, et le rythme stylistique des explications qui suivent: œil pour œil, dent pour dent (...) élèvent l'ensemble à un niveau supérieur. C'est de vie qu'il s'agit surtout, aussi bien pour l'animal que pour l'homme: humain pour humain, animal pour animal $^{26}$.

Ultimement, c'est un critère théologique qui fonde et dirige le travail de l'énonciation sur la sémantique de la langue, lui-même miroir de l'alchimie pratiquée par le Lévitique en son entier, dans la suite de la création-séparation de Genèse 1,2 et 3 . À tous les niveaux du texte, l'acte de lecture assiste à une opération de séparation, après le jardin en Éden l'enclos de la Loi, de décantage et densification de l'intégration réciproque de Yahvé et des humains, souvent à travers

26. Quant à la mise à mort des animaux pour abattage pour fins sacrificielles ou alimentaires, Lv 17,1-16 et Dt 12,13-28 en réglementent scrupuleusement les conditions, les lieux, les modalités et le respect dû au sang versé. 
le moyen terme des animaux. Le législateur forge et affine le peuple de l'Alliance. C'est «le passage au noir» du Grand CEuvre et de l'Alchimiste lui-même.

Une étude plus élaborée de la situation des animaux dans l'Ancien Testament se devrait de toucher à la critique des sacrifices chez les prophètes, jusqu'aux derniers versets du livre de Zacharie où, «à ce jour-là ", les grelots des chevaux et toute marmite à Jérusalem et en Juda porteront l'inscription «consacré au Seigneur» (Za 14,2021), si bien que la sacralisation du pays entier annulera la démarcation entre sacré et commun, pur et impur. L'étonnante ânesse de Balaam, prophétesse quadrupède, nous pardonnera de la délaisser elle aussi et de nous en tenir à cette fenêtre ouverte sur l'avenir des animaux par un des plus beaux passages du livre d'Isaïe (11,6-9). "Quand la connaissance de Yahvé remplira le pays », plus de lions et de bêtes féroces sur les chemins (Is 35,9); ils cohabiteront en toute amitié avec les animaux de la ferme, loup et agneau, panthère et chevreau, lionceau et veau, vache et ourse. Ils savoureront à nouveau les conditions de vie du paradis retrouvé, herbivores avec les ruminants et aimables compagnons de jeu des plus vulnérables des humains, jeunes enfants et nourrissons. Même l'inimitié mortelle entre vipère et descendance de la femme aura pris fin. Dans son télescopage de la temporalité, une vision de futur antérieur ${ }^{27}$.

\section{Les animaux sous la Nouvelle Alliance. De Jésus, à Pierre, à Jacques et à Paul}

Narrativement, la population animale des livres du Nouveau Testament descend de la souche vétérotestamentaire qui a survécu au

27. Vision prophétique quant au sort des animaux ou imagerie bucolique parfaite pour dire la paix et la non-agressivité? Le rapprochement avec Gn 3,15 à propos des relations entre les lignages de la femme et du serpent est saisissant, mais il joue entre un discours dit prophétique et un discours mythique. La question demeure sans réponse, à mon avis tant que nous ne posséderons pas de travaux sur la production et l'utilisation de la métaphore propre au discours prophétique, ce qui équivaut à soulever la question plus large de ses caractéristiques en typologie des discours. 
déluge. Figurativement, la population israélite de ces mêmes livres la perçoit à travers les caractéristiques attribuées dans l'Ancien Testament, c'est-à-dire composée d'êtres de la classe des vivants ${ }^{28}$, répertoriés selon leur habitat aquatique, aérien et terrestre, surdéterminés selon le pur/impur dans leurs deux fonctions alimentaire et sacrificielle. De plus, les animaux sont des organismes pourvus de sang, comme les humains, un sang frappé d'interdiction de consommation. «Car la vie de toute créature, c'est son sang ( $\operatorname{Lv} 17,1-15)$, « et tu ne mangeras pas la vie avec la viande» (Dt 12,23 à l'intérieur du passage 13-28). Dans le cas de la bête abattue pour fins alimentaires, le sang est répandu sur le sol et recouvert de terre; le sang de la victime sacrificielle est versé ou aspergé sur l'autel ou appliqué sur les personnes et les choses à purifier ou à consacrer. «Et moi je vous l'ai donné ce sang pour faire sur l'autel le rite d'expiation (pardon, absolution, purification) pour vos vies (pour vous), car c'est le sang qui réalise l'expiation parce qu'il est la vie» $(17,11)$. Le sang est formellement réservé aux rites de purification et de revivification du lien de l'Alliance. Dans un système sacrificiel qui proscrit le sacrifice humain, ce don de Dieu, c'est donc le sang des animaux.

Ce statut particulier de la gent animale aura un impact inattendu après le passage de Jésus. À l'arrivée de convertis venus du paganisme, les judéo-chrétiens devront prendre position devant ce volet de leur héritage mosaïque ancré dans leur vision du monde et les replis les plus intimes de la trame de leur vie. Le sort de l'expansion du christianisme va se jouer, pour une part, en lien avec celui des animaux. Au lieu de suivre ces derniers à la trace, comme pour l'Ancien Testament, nous les rechercherons plutôt chez des acteurs majeurs du Nouveau, dans leurs rapports aux prescriptions lévitiques. De Jésus, à Pierre, à Jacques et à Paul.

28. Dans le grec du texte, animal se dit zoon (vivant) ou psuchè zoês (être vivant) ce qui correspond exactement à l'hébreu nefesh hayyāh. 


\subsection{Jésus}

Il ne serait pas sans intérêt de tenter de cerner l'attitude du personnage Jésus envers les animaux. Cependant, les rédacteurs du Nouveau Testament se sont davantage intéressés aux animaux métaphoriques et emblématiques qu'aux animaux du monde naturel. D'autres types d'exploration du texte restent possibles. "Jésus avait-il un chien? ", question intéressée de Snoopy, le penseur canin du bédéiste Schulz. Combien d'animaux traversent ses discours... le chameau et le chas de l'aiguille, les serpents et les colombes en affaires, les renards et leurs tanières, le bœuf et l'âne menés au puits le jour du sabbat, les oiseaux du sermon sur la montagne nourris par le Père, la poule qui rassemble ses poussins, tant et tant de brebis... ? Le nombre d'occurrences et la finesse de l'imagerie nous en apprendraient pourtant moins sur Jésus et les animaux que deux épisodes des évangiles lourds de conséquences pour eux, puisqu'ils touchent à leurs deux fonctions séculaires.

Une controverse avec les Pharisiens et quelques scribes, amorcée par l'omission du lavement rituel des mains avant le repas par certains disciples de Jésus, provoque chez lui une réaction vigoureuse (Mc 7,1-23 ; Mt 15,1-12). Prendre son repas avec des mains impures (littéralement: "communes " par opposition à sanctifiées, de koinoô, rendre commun) transgresse la tradition des anciens, disent-ils. La réponse série les niveaux de tradition: la tradition des anciens, rajoutée à celle de Moïse, n'est que tradition des hommes par rapport au commandement de Dieu, n'est que la vôtre. Vous occultez la parole de Dieu par cette tradition que votre parole transmet.

Au bénéfice de la foule qu'il appelle expressément, Jésus élève le niveau de discussion cette fois. «Écoutez-moi tous et comprenez! Il n'y a rien d'extérieur à l'homme qui puisse le rendre impur en pénétrant en lui, mais ce qui sort de l'homme, voilà ce qui rend l'homme impur. "Une fois rentrés à la maison, les disciples obtiennent un éclairage complémentaire, sans équivoque. Ce qui est ingéré de l'extérieur ne pénètre pas dans le cœur de l'homme mais dans son ventre, puis s'en va aux lieux d'aisance. C'est tout le mal qui sort du cœur de l'homme qui le rend rituellement impur (littéralement: 
«commun»): desseins pervers, débauches, vols, meurtres, cupidité... la liste est longue et se termine par orgueil et déraison.

Des mains au cœur, c'est le parcours de la citation d'Isaïe (v. 6-7) dénonçant le faux culte qui ne passe pas des lèvres au cœur. Mais Jésus en Marc ne revient pas aux mains inappropriées causes de litige, comme il le fait dans le parallèle de Matthieu 15,1-20. Il conserve à sa réponse l'ampleur qu'il a voulu lui donner. Si bien que l'énonciateur s'interpose à la fin du v. 19, pour que le lecteur ne perde rien des retentissements de la séquence: "Il déclarait ainsi que tous les aliments sont purs $^{29}$. Relu dans le cadre global du Nouveau Testament, le discours de Jésus signifiait, en effet, à ses auditeurs l'abrogation des interdits alimentaires et, quant aux animaux, la fin de leur classification en purs et impurs. Et Jésus confirme sa prise de position orale par sa pratique de la communauté de table avec des Israélites légalement impurs de par leurs métiers, publicains et prostituées. Consommait-il également des animaux impurs? Les évangélistes n'en soufflent mot.

Question plus épineuse, l'épisode vise-t-il à montrer la priorité de l'impureté morale sur l'impureté légale, comme on le lit dans certains commentaires sérieux ? Oui et non. Le résultat du mal moral n'est mis en lien qu'avec l'impureté rituelle; ce mal rend l'homme impropre au culte, "commun" $(7,23)$, comme les mains non lavées sont «communes» $(7,2)$. En outre, si l'on tient compte de la finale du v. 19 qui a valeur de clé de lecture, l'impureté légale et des aliments et de leurs consommateurs tombe en plus d'être reclassée. Or, l'impureté de la non-observance de certaines ablutions relevait bien de la seule tradition ajoutée par les anciens, mais celle de certains aliments remonte au Lévitique considéré, lui, comme commandement de Dieu. L'herméneute Jésus renvoie lecteurs et exégètes à l'observation du texte et leur laisse comme tâche la mesure de la portée de sa relecture du Pentateuque.

Un deuxième épisode montre Jésus en relation avec l'animal sacrificiel au moment de l'incident que nos bibles intitulent « $\mathrm{La}$

29. Litt. : rendant (ainsi) purs (katharizôn) tous les aliments. 
purification du Temple» (Jn 2,13-22; Mc 11,15-17; Mt 21,12-17; Lc 19,45-46). À Jérusalem pour la Pâque proche, il trouve dans la cour extérieure du Temple les marchands de bœufs, de brebis et de colombes et les changeurs pour les offrandes monétaires. S'étant fait un fouet de cordes, il les chasse tous, hommes et bêtes, ordonne aux marchands de colombes d'emporter leurs cages, renverse les tables des changeurs. "Enlevez tout cela d'ici! Ne faites pas de la maison de mon Père une maison de trafic, une caverne de bandits. » Le récit de cette colère concerne d'abord Jésus dans la suite des prophéties de Zacharie et de Malachie ${ }^{30}$, et face à sa mort. Je ne crois pas que le fouet brandi témoigne de sa brutalité envers les animaux et qu'il vaille même la peine de le justifier en l'opposant à la sollicitude du pasteur pour ses brebis attribuée à Jésus à pleines pages des évangiles. Une lecture de la scène en contexte y suffit, et devrait suffire à redresser des opinions courantes diffusées par les médias.

L'épisode trouve plutôt sa véritable résonance dans l'épître aux Hébreux; elle nous en apprend davantage sur le futur de la fonction sacrificielle dévolue aux animaux que leur expulsion du Temple, visant d'ailleurs leurs mercantiles propriétaires. Jusqu'ici la Bible nous a représenté cette fonction en discours narratif dans la Genèse, puis législatif au Lévitique. Hébreux la transpose, avec la mort de Jésus, en registre métaphorique; l'épître opère ce que le langage du texte appelle une double métathèse. Un premier degré de métaphorisation transpose dans la configuration sacrificielle d'Israël le récit, non rituel, de la mort de Jésus au Golgotha. Un deuxième degré porte le récit sacrificiel au-delà du régime d'alliance qui le régissait; il le dépouille du sacrificiel maintenant aboli avec lui, subsumé dans la nouvelle alliance.

Les chapitres centraux établissent une comparaison terme à terme - sacrificateurs, victimes, effet du sacrifice - entre les sacrifices relevant de l'Alliance mosaïque codifiés au Lévitique et le sacri-

30. Za 14,21: et il n'y aura plus de marchand dans la maison de Yahvé Sabaot en ce jour-là. Ml 3,1 : Subitement, il entrera dans son Temple, le Seigneur que vous cherchez; l'Ange de l'Alliance que vous désirez, le voici qui vient, dit Yahvé Sabaot. 
fice de Jésus ratifiant l'Alliance nouvelle et éternelle. Au premier degré de métathèse, le sang des veaux et des boucs, des taureaux et des génisses $(9,12-14)$, utilisé par Moïse au Sinaï (9,18-22), offert chaque jour, sans cesse, et dont le grand-prêtre se munit pour entrer dans le sanctuaire une fois l'an. Au deuxième degré, celui de la mort de Jésus, son propre sang, répandu une seule fois, une fois pour toutes $(9,25-28 ; 10,14 ; 7,27)$. La comparaison entre les deux régimes, magistralement menée à fleur-de-texte et en structure profonde par l'ensemble de l'épître, aboutit à déclarer la mort de Jésus moment de passage entre l'ombre et la réalité ${ }^{31}$. Le point d'arrivée définitif par accession à une Alliance plus parfaite met fin au système sacrificiel d'Israël, aussi sûrement que l'action des soldats de Titus détruisant le Temple de Jérusalem en l'an 70. L'épître aux Hébreux est aussi l'annonce de la fin de la servitude des animaux sur ce plan, la Bonne Nouvelle proclamée à la gent animale.

Dans le récit évangélique, la prise de position de l'acteur Jésus devant la catégorie pur/impur et les déviations du culte est d'une telle force et d'une telle clarté qu'on en oublie la distance qu'elle ouvrait dans le personnage lui-même par rapport aux coordonnées spatio-temporelles, à la formation et l'orientation religieuse ambiante à lui attribuées par le texte. Les metanoïa individuelles et communautaires des premiers chrétiens ne connaîtront pas le même rythme d'évolution. Sauf chez Paul, elles vont osciller, à des degrés divers, avant de trouver leur assise.

\subsection{Pierre}

De même extraction géographique, ethnique et sociale que Jésus, sensiblement de la même génération, il a vécu dans son intimité et consacre sa vie à poursuivre son œuvre. Pourtant, une extase devra

31. Pour le détail de l'analyse, voir O. Genest, Le discours du Nouveau Testament sur la mort de Jésus. Épîtres et Apocalypse, Sainte-Foy, Les Presses de l'Université Laval et la Corporation Canadienne des Sciences Religieuses, 1995, p. 124-156. Des éléments essentiels à cette lecture d'Hébreux n'ont pu trouver place dans cet article. 
lui apprendre ce qui lui avait échappé de l'enseignement de son Maître et de sa pratique. À Joppé, sur la terrasse de la maison de son hôte, à midi, une sensation de faim trouble sa prière. Le ciel s'ouvre et une toile immense suspendue par ses quatre coins descend vers la terre. Sur cette toile, tous les animaux quadrupèdes, tous ceux qui se meuvent sur la terre et ceux qui volent dans le ciel. Une voix lui dit: «Allons, Pierre, tue et mange!» «Jamais, Seigneur, répond Pierre, car de ma vie je n'ai mangé quoi que ce soit d'interdit (litt. de «commun", de non conforme à la pureté rituelle) ni d'impur!» La voix reprend: "Ce que Dieu a rendu pur, tu ne vas pas, toi, le déclarer interdit!» (Ac 10,9-16)

Le scénario se répète trois fois. Pierre n’y comprend rien, mais des cris se font entendre au portail de la maison et l'esprit lui enjoint de suivre ces messagers à Césarée, chez Corneille le centurion romain. La vision s'éclaire: "à moi Dieu vient de m'apprendre qu'il ne fallait déclarer interdit ou impur aucun être humain » (v. 28). Des animaux aux humains, à la communauté de table, de vie et d'union à Dieu qui s'ensuit. Le don de l'Esprit suivi du baptême ratifie la compréhension de Pierre et son évangélisation de la maison de Corneille.

Pierre à Jérusalem

Deux séries concomitantes de manifestations divines accordées au centurion Corneille et à Pierre, couronnées par la Pentecôte de Césarée, ont installé ce dernier dans une prise de position ferme par rapport à l'entrée des païens dans la communauté chrétienne. Il aura à la justifier lors d'un passage à Jérusalem (Ac 11,1-18). Des croyants venus de la circoncision le prirent à partie. "Tu es entré chez les incirconcis et tu as mangé avec eux! » Sous l'accusation de mépris de la Loi et de triple impureté, commerce avec des incirconcis, séjour chez eux et communauté de table, Pierre reprend élément par élément le récit des événements de Joppé-Césarée. Il ajoute les bêtes sauvages, soit les animaux de «l'ailleurs", à la description de sa vision, détail qui cadre bien dans un plaidoyer pour l'extérieur de "l'ici» d'Israël. Le calme revient et les contestataires font le pas décisif de Pierre: «Voilà que Dieu a donné aussi aux nations païen- 
nes la conversion qui mène à la vie! » Mais ils n'en ont pas encore mesuré toutes les implications. Et Pierre non plus.

\subsection{Paul}

L'acteur Pierre incarne le point de vue de l'Israël géographique, de la Judée surtout. La question de l'accueil des pagano-chrétiens s'y pose de façon aiguë. Faut-il les considérer comme des immigrants étrangers, leur donner le statut de craignants-Dieu, de ces non-Juifs séduits par la foi d'Israël et s'adonnant à quelques pratiques de la Loi, ou leur imposer celui de prosélytes avec circoncision et observance obligatoires. En plus de toutes les solutions à inventer au cours d'un quotidien régi dans ses moindres détails — ne recourt pas qui veut aux interventions célestes - la question soulève rien de moins que le lien entre christianisme et yahvisme. Par contre, pour l'acteur Paul, Juif né, éduqué et vivant en diaspora avec les privilèges de citoyen romain de naissance, le problème d'appartenance territoriale des païens convertis au Christ ne se pose même pas. Par une de ses évolutions foudroyantes dont témoigne sa correspondance personnelle, il a de plus dépassé sans retour la barrière du pur/impur, tout débordant de zèle pour la tradition de ses pères qu'il était d'abord, "ne respirant que menaces et meurtres » contre les disciples de Jésus (Ac 9,1).

\section{Paul à Jérusalem}

Le citoyen de Tarse s'approchant de la communauté chrétienne venait doublement de l'extérieur. Il n'avait pas connu Jésus, n'était pas l'un des Douze et il se présentait comme l'apôtre des incirconcis, de par la grâce de Dieu. Lors d'une brève rencontre à Jérusalem, il fit la connaissance de Céphas (Pierre) et de Jacques «le frère du Seigneur $^{32} »$. Quatorze ans plus tard, Paul juge bon de faire accréditer

32. L'autre Jacques, frère aîné de Jean, fils de Zébédée, a déjà été décapité sur les ordres d'Hérode Agrippa I ${ }^{\text {er }}$, entre les années 41 et 44. 
son apostolat et revient à Jérusalem exposer l'évangile qu'il prêche aux païens (Ga 2,1-10). Les «colonnes de l'Église », Jacques, Céphas et Jean, reconnaissent sa mission auprès des incirconcis comme celle de Pierre auprès des circoncis, lui donnent la main ainsi qu'à Barnabas en signe de communion, ne contraignent aucunement leur compagnon le Grec Tite à la circoncision. Une seule consigne: "Simplement, nous aurions à nous souvenir des pauvres.» Pas plus que Pierre dans l'enthousiasme de Césarée, Paul ne prévoyait les rebondissements futurs.

\subsection{Pierre, Paul et les gens de Jacques à Antioche}

Les accords de Jérusalem ne tiennent pas longtemps la route. Jacques exerce le rôle prépondérant dans l'église de la Ville sainte et des judéo-chrétiens de son entourage descendent à Antioche y relancer la controverse de l'imposition de la circoncision. À leur arrivée et devant la virulence des affrontements (Ac 15,1-3; Ga 2,12-14), la position de Pierre vacille; il se dérobe, cesse de prendre ses repas avec les païens entraînant dans sa duplicité les frères juifs, et même Barnabas. Paul l'accuse devant tout le monde de ne pas marcher selon la vérité de l'Évangile. "Si toi qui es Juif, tu vis à la manière des païens et non à la juive, comment peux-tu contraindre les païens à se comporter en Juifs?»(Ga 2,14-15)

\section{Pierre, Paul et Jacques à Jérusalem}

Le conflit d'Antioche dut être porté devant les apôtres et les anciens à Jérusalem (Ac 15,5-35). Cette fois plus nuancé et plus réaliste, le jugement de Jacques tient compte des deux factions, celle des fidèles issus du pharisaïsme et celle de Pierre, de Paul et Barnabas. "Je suis d'avis de ne pas accumuler les obstacles devant les païens qui se tournent vers Dieu. Prescrivons-leur simplement de s'abstenir des souillures des idoles (c'est-à-dire de ce qui a servi au culte des idoles), de la porneia, de la chair d'animaux étouffés (donc non saignés) et du sang. » La lettre que porteront deux délégués de l'assemblée réaccompagnant Paul et Barnabas à Antioche contient les mêmes 
termes sous l'en-tête: «L'Esprit Saint et nous-mêmes avons décidé de ne vous imposer aucune charge que ces exigences inévitables.»

Si l'on traduit porneia par "unions impudiques, illégitimes", sur l'arrière-plan de Lv 18,6-18, la liste des prescriptions s'unifie: elle est manifestement de l'ordre de la pureté rituelle ${ }^{33}$. L'habileté de la formule, c'est qu'elle recouvre à peu près les sept préceptes de l'alliance avec Noé, tels que comptabilisés dans la littérature rabbinique, préceptes qui s'appliquaient à toute l'humanité avant la distinction entre Juifs et Gentils. Le pôle Jacques-Jérusalem avait troqué l'obligation de la circoncision et de l'allégeance mosaïque complète contre l'assurance de la pureté légale minimale, rendant possibles la communauté de table et les eucharisties entre judéo et pagano-chrétiens. Le pôle de Pierre-Paul avait reculé par rapport à Joppé-Césarée, mais le christianisme naissant pouvait respirer et vivre.

Quant aux animaux, présents à leur insu dans trois des quatre prescriptions du décret salvifique, ils pouvaient aussi mieux respirer. En régime chrétien, la classification zoologique selon le pur/impur s'évanouissait. Un animal non saigné ou intégré au culte des fauxdieux était toujours impur et source d'impureté, selon Jérusalem I, mais cela dans son lien au sang ou à l'idole et non plus selon ses caractéristiques somatiques propres avant toute insertion dans les processus sacrificiels et alimentaires.

33. Si par ailleurs, sous l'influence de la variante occidentale du texte des Actes, on traduit eidôlôn par idolâtrie, porneia par immoralité et qu'on tient compte de l'ajout de la règle d'or "ne pas faire à autrui ce qu'ils ne voudraient pas qu'il leur soit fait à eux-mêmes » (Mt 7,12) ce qui conduit à rendre sang par homicide, on moralise l'ensemble du décret. Or, l'abandon de l'idolâtrie était déjà sous-entendu dans la conversion des pagano-chrétiens, et cette conversion n'est nullement mise en doute, mais la présence du culte des idoles, elle, perdure à tous les niveaux de leur vie socio-politique. Et la question qui avait provoqué ce premier concile de Jérusalem, comme on se plaît à l'appeler parfois, n'était pas d'ordre moral mais bien plutôt: que devons-nous exiger des hellénoromano-chrétiens pour que les judéo-chrétiens, toujours sous la loi de Moïse, puissent les fréquenter sans souillure légale. 
Néanmoins, la règle du pur/impur demeurait en vigueur à propos des humains. Les contacts entre convertis issus du judaïsme et convertis venus du paganisme comportaient toujours la menace de souillure par contamination. De là le maintien de ces «exigences inévitables ", de l'ordre de la nécessité, de l'ordre des choses. Comment l'auguste assemblée de Jérusalem avait-elle pu ne pas comprendre le langage clair de Joppé-Césarée? De la même façon, sans doute que de bénignes églises du $\mathrm{xxI}^{\mathrm{e}}$ siècle cultivent encore de cruelles, «d'inévitables» ( ?) marginalisations.

\subsection{Paul dans ses Lettres}

Sauf dans quelques versets de l'épître aux Galates, c'est le Paul selon Luc, au livre des Actes, que la lecture a rencontré jusqu'ici. Paul selon Paul, dans ses lettres, rejoint la position claire et ferme de Jésus, en deçà et bien au-delà de la solution d'appoint élaborée à Jérusalem. Il aura à l'exposer à ses correspondants à propos de la consommation d'animaux immolés cette fois à d'autres cultes que celui de Yahvé. Cette question dépasse la réglementation d'une pratique; elle ne peut trouver sa casuistique et sa théorie que dans l'élaboration d'une conception du pur/impur, quelle qu'elle soit.

La réflexion de Paul s'appuie sur ce qui est devenu pour lui un plancher d'évidences, là où d'autres chrétiens s'enferrent encore à des degrés divers. L'idole non seulement n'a aucune valeur, mais elle n'est rien dans le monde (1Co 8,4-5.10-19). "Ce n'est pas un aliment qui nous fera comparaître en jugement devant Dieu. Si nous n'en mangeons pas, nous n'avons rien de plus; et si nous en mangeons, nous n'avons rien de moins. » $(8,18)$ «Tout ce qui se vend sur le marché, mangez-le sans poser de question par motif de conscience car la terre est au Seigneur et tout ce qui la remplit.»(10,25-26) "Car le Règne de Dieu n'est pas affaire de nourriture ou de boissons. » (Rm 14,17) «J'en suis convaincu dans le Seigneur Jésus, rien n'est impur (koinòn) en soi. Mais une chose est impure pour celui qui la considère telle.» $(14,14)$ «Dès lors que nul ne s'avise de vous critiquer sur des questions de nourriture et de boisson [...] $\mathrm{Ne}$ prends pas, ne goûte pas, ne touche pas, toutes (ces ordonnances) 
pour des choses vouées à périr par leur usage même!» (Col 2,16. 21-22) Enfin, cette formule lapidaire, tirée d'une épître deutéropaulinienne mais faisant écho à $\mathrm{Rm}$ : «Tout est pur pour les purs.» (Ti 1,15)

Cependant, les nouveaux convertis du paganisme, quelles que soient leurs convictions ou leurs hésitations, n'échappent pas à la présence constante des idolothytes ${ }^{34}$ dans leur vie. Même si eux ont rompu avec l'idolâtrie, le surplus des viandes consacrées aux idoles débordent de l'intérieur des sanctuaires vers les salles-à-manger des dépendances des temples, le marché public, les maisons privées. Elles servent et on les sert aux repas de mariage, d'anniversaire, de funérailles, de partenariat commercial, de fêtes de la cité. Pour Paul, la discussion des situations embarrassantes provoquées par leur omniprésence se résout à un plan jugé supérieur et prioritaire; il s'en explique dans les remarquables chapitres de 1Co 8-10 et de Rm $14,1-15,13$.

La libération dans le Christ ne libère pas d'une prudence élémentaire à l'égard de pouvoirs occultes qu'on ne maîtrise pas. S'asseoir à une table, parmi les convives, instaure un réseau de relations, $\mathrm{y}$ compris avec la divinité dans le cas d'un rite cultuel. Attention donc à l'entrée en communion à laquelle on s'expose dans la consommation de mets provenant des sacrifices. L'idole n'est rien, l'animal sacrifié que de l'honnête viande, mais les sacrifices des idolâtres sont offerts aux daimonia ${ }^{35}$, qui eux sont bien réels selon Paul. "Vous ne pouvez boire à la fois à la coupe du Seigneur et à celle des démons;

34. Du grec eidôlothuta, de eidôlon: image, idole et thyô: sacrifier, immoler. À cause du verbe immoler, on traduit par: les animaux, les viandes sacrifiés aux idoles. Avec l'usage, le référent de tuer en sacrifice s'est étendu à sacrifier, puis à offrir, et celui d'animal à tout autre nourriture offerte dans les rites cultuels, huiles, miels, vins, grains, farines, fruits.

35. Les Corinthiens auxquels il s'adresse ne s'y retrouveraient sans doute pas tout à fait dans les connotations du mot "démons" de nos traductions. Pour eux, les daimonia sont de l'ordre des theoi, des dieux et, précise Paul en 8,5: «de fait il y a quantité de dieux et quantité de seigneurs " soit au ciel, soit sur la terre. 
vous ne pouvez participer à la fois à la table du Seigneur et à celle des démons. » $(10,21)$

Les néo-chrétiens ne peuvent néanmoins s'extraire de leur situation familiale et sociale; elle comporte des obligations conviviales incontournables. Le respect de l'hôte prime ici. "Si un non-croyant vous invite, et que vous acceptiez d'y aller, mangez tout ce qui vous est offert sans poser de questions pour motif de conscience. Si quelqu'un, par motif de conscience, la sienne, vous prévient: "C'est de la viande sacrifiée", respectez ses scrupules et abstenez-vous.» (1Co 10,27-29). La libération dans le Christ comporte des degrés et des rythmes de croissance différents. "La foi de l'un lui permet de manger de tout, tandis que l'autre, par faiblesse, ne mange que des légumes. Que celui qui mange ne méprise pas celui qui ne mange pas et que celui qui ne mange pas ne juge pas celui qui mange, car Dieu l'a accueilli. » (Rm 14,2-4) Entre le Juif, le Grec, les Puissances d'une cosmologie protéiforme et l'Église de Dieu, le barème suprême de discernement devient le respect du frère «pour lequel le Christ est mort» (Rm 14,15).

Au carrefour de toutes ces instances, l'animal est ramené à ses propres dimensions, ni magiques, ni méprisables. Comme Isaï, Paul ouvre une fenêtre sur son avenir, dans un discours construit, cette fois, de figures d'ordre cosmique, tissées d'un vocabulaire de rédemption en lier/délier devenu «technique » autour de la mort de Jésus (Rm 8,19-25).

Au temps primordial de Genèse 3, la création a été assujettie, liée à la vanité, au statut qui n'a pas de cohésion véritable et qui aboutit à la mort. Par la volonté de qui l'y a soumise, contre son désir mais dans l'espérance d'être, elle aussi, libérée de l'esclavage de la corruption et promise à l'entrée dans la liberté de la gloire des enfants de Dieu. "Nous savons, en effet", écrit Paul, "que toute la création ensemble-gémit» ou «que toute la création tout entière (ce qu'il exprime par le préfixe sun: avec, apposé au verbe) est ensemble-entravail d'enfantement jusqu'à ce jour. Nous aussi, qui avons pourtant déjà les prémices de l'esprit, nous gémissons (le préfixe de l'unanimité a disparu) dans l'attente de l'apolutrôsis, c'est-à-dire de 
la rédemption, de la délivrance de notre condition somatique ${ }^{36}$ actuellement soumise à la loi de la corruption. Et l'esprit lui-même se joint à cette attente haletante en intercédant pour nous avec des "gémissements inexprimables» (v. 26).

Pour les animaux livrés aux mains des humains au sortir de l'arche de Noé, liés au lourd service de l'alimentation des humains et des dieux au prix de leur vie, la vision cosmique paulinienne, qui les incorpore dans l'usage du préfixe sun, signifie la restauration, la réintégration totale. Sans codicille les excluant, le texte leur promet plus encore, soit la délivrance de la loi de la mortalité des vivants. La création entière attend avec impatience la révélation de la gloire propre au statut d'enfants de Dieu, qui sera manifestée à la pleine réalisation d'un salut encore à l'état de prémices. Nous pouvons aussi nous permettre d'attendre avec beaucoup de curiosité la manifestation de ce qui apparaîtra dans la rédemption des animaux.

\section{Bilan de lecture. La situation des animaux dans la bible}

Les animaux apparaissent au cinquième jour de la création, premiersvenus de la catégorie des êtres vivants. Ils en portent le nom, en cela mieux nantis qu'Adam «la créature tirée du sol», le terreux. Dans sa traduction du grec de la Septante, Marguerite Harl écrit à ce cinquième jour: «Et Dieu dit: Que les eaux fassent sortir celles des âmes vivantes qui sont des reptiles et des volatiles [...]» et au sixième jour: "Que la terre fasse sortir l'âme vivante selon son espèce, quadrupèdes [...] selon leur espèce ». Si le grec n'avait pas varié le style, nous aurions pu lire à propos de l'homme: «Faisons celles des âmes vivantes qui sont des humains ». Ces formulations font

36. Litt.: la délivrance pour notre corps. Je traduis par condition somatique pour éviter l'ambiguïté de l'opposition corps/âme dans une pensée qui ne la comporte pas et qui ferait croire à une rédemption en deux étapes, celle de l'âme puis celle du corps. Ph 3,20-21 décrit cette libération somatique comme la transformation de notre actuel corps de misère en sa conformité avec le corps de gloire du Seigneur Jésus. 
ressortir la parenté entre les vivants posée par les contextes, le lien qui fonde la valeur et la dignité de l'animal ${ }^{37}$.

L'animal est mis en discours comme détenteur du souffle et du sang caractéristiques de sa catégorie, placé sous la gérance des humains dans la hiérarchie des vivants, livré entre leurs mains après le déluge mais avec l'annonce de la libération de sa longue servitude aux temps messianiques selon certains passages de la littérature prophétique. Sa diversité figurative débordante est classifiée d'abord selon les espaces par lui habités, mer, ciel et terre. D'un livre à l'autre, quelle que soit sa forme littéraire, le texte reprend avec des variantes vraiment mineures la nomenclature vite stéréotypée de: poissons, oiseaux, bêtes sauvages ou des champs, bestiaux, petites bêtes du sol. Elle sera elle-même très tôt traversée en chacun de ses groupes par la sur-qualification du pur et de l'impur. Viendra s'y ajouter une classification encyclopédique d'après les organes de locomotion, également régie par le pur/impur, c'est-à-dire, quant à l'animal, par le propre-à/impropre-à l'accomplissement de ses deux fonctions dans le cadre de l'Alliance: servir de nourriture conforme à l'état de pureté rituelle du peuple séparé par et pour Dieu, alimenter le culte du Tout-Autre ${ }^{38}$. En ce sens, celui de la loi de pureté, du Code de perfection (Lv 11-16), l'animal jugé pur pourra être dit «chose sainte », chose séparée pour Dieu.

37. Quant à l'ambiguïté de l'emploi du mot âme en littérature sémitique, la traductrice s'en explique à partir du choix, par les Septantes eux-mêmes, du mot psuchè comme équivalent fixe de l'hébreu nefesh hayyāh. La discussion de son option personnelle nous vaut un condensé remarquable des acceptions du mot âme à partir de l'hébreu original en passant par le grec, les exégèses platonisantes de Plotin et des Pères grecs jusqu'au français. Voir C. Dogniez et M. Harl, dir., Le Pentateuque. La Bible d'Alexandrie (Folio/Essais 419), Paris Gallimard, 2001, p. 40-41; 813-815.

38. Parmi les multiples instructions du Lévitique, il en existe une troisième: fournir le sang des rites de purification qui opèrent hors liturgie la réintégration des personnes ou de leurs biens après rupture d'avec le tissu communautaire par contamination involontaire. Exemple: la purification d'un lépreux guéri (Lv 14,1-32) ou d'une maison atteinte de la lèpre (14,33-57). 
Le Nouveau Testament modifie totalement le statut biblique de l'animal. La prise de position radicale de Jésus contre la ségrégation du monde et de ses habitants selon le pur et l'impur le renvoie, comme l'humain d'ailleurs, à l'emploi absolu de sa dénomination de «vivant» en grec comme en hébreu. Elle le rend à sa valeur propre et le relève de sa fonction de purification au prix de son sang. $\mathrm{La}$ formulation métaphorique de l'épître aux Hébreux va plus loin: le sang de la mort de Jésus répandu une seule fois, une fois pour toutes, met fin à l'effusion de celui des animaux offerts chaque jour et sans cesse dans le cycle sacrificiel de l'Alliance mosaïque.

Certains acteurs des Actes et des épîtres incarnent textuellement les divers degrés d'absorption de l'onde de choc déclenchée par les déclarations de Jésus chez les judéo-chrétiens d'abord, puis chez les pagano-chrétiens et surtout à propos des conditions de possibilité de relations entre les deux groupes. Dans la question des viandes consacrées aux idoles et de la communauté de table et de vie, les animaux ont fait les frais des enjeux majeurs. On les retrouve partout dans les déclarations tâtonnantes des accords de Jérusalem, les attitudes et pratiques flottantes de Pierre, de Jacques et de Barnabé. Seul Paul maintient sans broncher le cap de l'innovation magistrale de Jésus, dans la ligne du mouvement de désacralisation du cosmos inaugurée par le texte de la Genèse. Ses phrases de Rm 8,19-25 ne sont donc pas une surprise sous sa dictée quand il montre, outre les humains, la création tout entière, dont les animaux donc, en travail d'enfantement de sa propre délivrance de la loi de la corruption.

$\mathrm{Au}$ sortir de ce survol selon le parcours choisi, nos questions de départ trouvent d'elles-mêmes leurs réponses. Les animaux de la Bible ne sont pas mis en discours comme de simples éléments d'un calque du réel. Ils ont une vie textuelle en tant qu'acteurs dotés de rôles thématiques et actantiels, d'une figurativité hautement diversifiée et d'un destin dont le lecteur peut suivre la trajectoire, y compris dans sa partie projetée dans le futur d'une histoire universelle. Il leur est promis une délivrance bien à eux et pas seulement le spectacle de celle de l'espèce humaine.

Les résultats de l'enquête ont-ils une utilité autre que la satisfaction de la curiosité de celle qui a voulu la mener à l'aide de ses outils 
d'exégète? De l'avis de l'auteure, leur premier acquis est de nous mettre sous les yeux l'ensemble de la situation des animaux dans la Bible, au-delà de la réponse par citation de versets tirés de leurs contextes médiats et immédiats. Le corpus biblique souffre dans l'opinion publique de l'impression de fausse familiarité qu'on en a. Combien d'études, et parfois des plus sérieuses, lues et entendues dans les médias, n'ont-elles pas pris l'habitude de parler de la dégradation du statut des animaux imputable au Nouveau Testament et au christianisme? Pour ma part, j'ai souhaité chercher l'origine de cette assertion, par souci de conformité au texte en deçà de l'attribution des responsabilités; je ne l'ai pas trouvée.

En revanche, le parcours adopté, en plus de signaler au passage l'ouverture d'autres avenues d'exploration, fait apparaître l'animal comme un élément bien défini d'une vision du monde relevant d'un groupe humain précis. Il est donné par le texte en état de système à l'intérieur d'un construit littéraire mythique véhiculant la cosmologie, l'anthropologie, la théologie, la sotériologie, l'historiographie religieuse de ce groupe. L'énonciation l'offre à la lecture à travers divers types de discours - narratif, prophétique, sapiential, hymnique, apocalyptique - au fil de livres de datations différentes étalées sur dix siècles de rédaction. De plus, l'ensemble nommé Bible peut être abordé à la fois comme un texte fixé par des frontières décrétées par des autorités religieuses ou en efflorescence dans des corpus rayonnants tant en littérature rabbinique qu'en productions chrétiennes.

De l'appartenance de l'animal biblique à ce grand tout textuel devra tenir compte quiconque ouvre le Livre pour y trouver un éclairage aux dilemmes éthiques de ce siècle. La réponse ne saurait être immédiatement transparente ni directement transposable dans notre vision du monde. Aux milliers de croyants pour qui la Bible est normative, la relation au Dieu qu'elle annonce suggérera plus sûrement les comportements justes envers les animaux que leur représentation biblique. Et faudrait-il encore être attentif au point de leur trajectoire que nos citations utilisent.

Les mêmes remarques s'appliquent à d'autres problèmes actuels dont on recherche la solution dans la Bible, contrôle de la reproduc- 
tion humaine, suicide, euthanasie, divorce, homosexualité, clonage, manipulations génétiques. La question la plus proche du statut biblique des animaux reste celle du statut des femmes du même corpus, statuts joints plus étroitement par le lien du pur/impur que par l'attribution d'échelons inférieurs dans les processus de hiérarchisation. Les animaux y ont plusieurs longueurs d'avance. Ils ont émergé de cette problématique, alors que, dans l'histoire des femmes, il manque encore aux trois grands monothéismes une vision de Joppé.

Plus globalement encore, une question en apparence modeste comme celle de la situation des animaux dans la Bible, posée en regard d'interrogations éthiques contemporaines, renvoie au lecteur individuel et collectif celle de sa situation devant cette Bible. Quelle est sa pratique de lecture d'un texte aussi complexe, qui opère à tant de niveaux? Le lecteur relu par les animaux bibliques! Peut-être même le surprendront-ils à rêver qu'au jour de leur rédemption selon Paul, des animaux réels, racontés jusque-là par les humains, se raconteront eux-mêmes et lui raconteront les humains ? L'humanité relue par les animaux.

\section{RÉSUMÉ}

Les discussions éthiques autour du comportement à adopter envers les animaux recourent souvent à des passages bibliques. Devant des réponses divergentes, l'auteure tente de faire le point sur leur statut dans la Bible, Ancien et Nouveau Testament. L'enquête porte sur les passages les plus significatifs: création, déluge, classification selon le pur et l'impur, rôle dans le système sacrificiel d'Israël et les cultes du monde gréco-romain où se répand le christianisme naissant. La conclusion soulève brièvement la question de la pertinence du recours à la Bible et de sa normativité sur ce point.

\section{ABSTRACT}

We frequently witness the use of biblical texts in ethical debates concerning the treatment of animals. In view of different responses, the author focuses on their status in the Bible, Old and New Testaments. The inquiry undertakes the most significant passages of Scripture: creation, flood, classification of clean and unclean, role in Israel's 
sacrificial system and in the cults of the Greco-roman world, birthplace of Christianity. In conclusion, the author briefly questions the use of the Bible as a guideline of how one should treat animals.

(C) Revue Théologiques 2002. Tout droit réservé. 